\title{
Further determination of DNA relatedness between serogroups and serovars in the family Leptospiraceae with a proposal for Leptospira alexanderi sp. nov. and four new Leptospira genomospecies
}

\author{
Don J. Brenner, Arnold F. Kaufmann, Katherine R. Sulzer, \\ Arnold G. Steigerwalt, Faye C. Rogers and Robbin S. Weyant
}

\author{
Meningitis and Special \\ Pathogens Branch, Division \\ of Bacterial and Mycotic \\ Diseases, Bldg 1-2226 \\ Mailstop D11, Centers for \\ Disease Control and \\ Prevention, Atlanta, GA \\ 30333, USA
}

\author{
Author for correspondence: Don J. Brenner. Tel: +1 404639 2841. Fax: + 14046394421 \\ e-mail:djb3@cdc.gov
}

DNA relatedness was determined among 303 strains of Leptospira and Leptonema. Included in the analysis were reference strains from 228 wellcharacterized and recognized serovars. The study included 268 serovars from 29 named and one or more unnamed serogroups. The strains clustered into 17 DNA hybridization groups, representing 12 previously described species (292 strains) and five new genomospecies (11 strains). The largest groups included Leptospira interrogans (91 strains from 82 serovars), Leptospira santarosai (65 strains from 59 serovars), Leptospira borgpetersenif (49 strains from 43 serovars), Leptospira kirschneri (29 strains from 26 serovars) and Leptospira noguchii (20 strains from 20 serovars). The new genomospecies include Leptospira genomospecies 1 (two strains, serovars pinagchang and sichuan), Leptospira genomospecies 2 (six strains, serovars lushui, manhao 3, manzhuang, nanding, mengla and yunnan), Leptospira genomospecies 3 (one strain, serovar holland), Leptospira genomospecies 4 (one strain, serovar hualin) and Leptospira genomospecies 5 (one strain, serovar saopaulo). With the exception of Ballum, all serogroups with greater than one serovar studied were genetically heterogeneous. Phenotypic tests, including optimal growth temperature, lipase activity and growth inhibition by copper sulfate or 2,6diaminopurine, were of little use in differentiating DNA relatedness groups. The name Leptospira alexanderi sp. nov. is proposed for Leptospira genomospecies 2 (type strain L 60' = ATCC 700520', serovar manhao 3 ).

Keywords: Leptospira, Leptonema, Leptospira alexanderi, Leptospira genomospecies, DNA relatedness

\section{INTRODUCTION}

Before 1979, the genus Leptospira contained two valid species, Leptospira interrogans and Leptospira biflexa. $L$. interrogans contained 23 serogroups whose strains were either parasitic or pathogenic for humans or animals (Johnson \& Faine, 1984; Kmety \& Dikken, 1993). L. biflexa contained 28 serogroups whose strains were usually found in fresh surface waters or moist soil and were rarely isolated from man or animals (Johnson \& Faine, 1984; Kmety \& Dikken, 1988).

As early as 1969, DNA relatedness studies on lepto- spires indicated that the 15 pathogenic and nonpathogenic serovars tested were contained in six DNA relatedness groups (Haapala et al., 1969; Brendle et al., 1974). Despite this finding of heterogeneity within both $L$. interrogans and $L$. biflexa, additional species were not proposed. In 1979, Hovind-Hougen proposed the new family Leptospiraceae, which comprised the genus Leptospira and the new genus Leptonema, with its single species, Leptonema illini (Hovind-Hougen, 1979), and in 1981, Hovind-Hougen and colleagues described a third Leptospira species, Leptospira parva (Hovind-Hougen et al., 1981). In 1992, the Subcommittee on the taxonomy of Leptospira decided that $L$. 
parva was sufficiently different from Leptospira and Leptonema to merit proposal as a new genus, 'Turneria' (Marshall, 1992). Since this genus was never formally described, it currently has no standing in nomenclature.

Heterogeneity within Leptospiraceae was again studied in 1986, when Yasuda et al. (1987) used DNA relatedness to compare 44 serovars from 23 recognized and three unnamed serogroups of $L$. interrogans and single serovars from $L$. parva and Leptonema illini. Their DNA relatedness studies confirmed the validity of the then new species, L. parva and Leptonema illini, and demonstrated that, as then defined, both $L$. interrogans and $L$. biflexa were extremely heterogeneous. They described seven new species: Leptospira borgpetersenii, Leptospira inadai, Leptospira noguchii, Leptospira santarosai, Leptospira weilii, Leptospira meyeri and Leptospira wolbachii. They further demonstrated that serogrouping did not strictly equate with speciation, since some serovars in different subserogroups within the same serogroup belonged to different species. Ramadass et al. (1992) described the most recent species, Leptospira kirschneri, in a study that included strains of 66 serovars.

The purpose of the present study was to further survey strains of leptospire serovars in order to properly speciate them and to better determine the species-level specificity of serogroups and serovars.

\section{METHODS}

The nomenclature of serogroups and serovars generally follows that of Kmety \& Dikken $(1988,1993)$. Serovars not included in the list of Kmety \& Dikken are shown in bold type in Table 1 . The methods used in this study for the cultivation of Leptospiraceae, for their biochemical characterization and for the determination of their DNA relatedness are essentially identical to those reported previously (Yasuda et al., 1987). These methods are summarized below.

Bacterial strains. Three-hundred-and-three strains were studied (Table 1). These organisms include representatives from 30 named and one or more unnamed serogroups in the genera Leptospira and Leptonema (Table 1). It has been the practice among leptospirologists to italicize serovars. We have not italicized taxa below the level of species. The term leptospires is used to refer to any members of the family Leptospiraceae.

DNA relatedness. Strains were grown at $30^{\circ} \mathrm{C}$ in polysorbate albumin medium and harvested by centrifugation during late-logarithmic or stationary growth. DNA was isolated and purified as previously described (Brenner et al., 1982). DNA was labelled in vitro with $\left[{ }^{32} \mathrm{P}\right] \mathrm{dCTP}$ (Brenner et al., 1982). DNA relatedness and percentage divergence within related sequences were determined by the hydroxyapatite method, with $55^{\circ} \mathrm{C}$ incubation used for optimal DNA reassociation and $70^{\circ} \mathrm{C}$ incubation used for stringent DNA reassociation (Brenner et al., 1982). Percentage divergence within related DNA sequences was determined to the nearest $0.5 \%$.

G $+\mathbf{C}$ content of DNA. The $\mathrm{G}+\mathrm{C}$ content ( $\mathrm{mol} \%$ ) was determined for DNAs from type strains of each of the five new genomospecies by the thermal denaturation method (Mandel et al., 1970).

Phenotypic characteristics. The following tests, as previously described, were performed on strains incubated at $30^{\circ} \mathrm{C}$ (unless stated otherwise) on polysorbate albumin medium: growth at 11,30 and $37^{\circ} \mathrm{C}$; growth in the presence of $225 \mu \mathrm{g}$ 8 -azoguanine $\mathrm{ml}^{-1}$ or in the presence of $10 \mu \mathrm{g}$ 2,6-diaminopurine $\mathrm{ml}^{-1}$, growth at $30^{\circ} \mathrm{C}$ in the presence of varying concentrations $\left(25,50,100,1000\right.$ and $\left.10000 \mu \mathrm{g} \mathrm{ml}^{-1}\right)$ copper sulfate; and the presence of lipase (trioleinase) activity. Growth was measured by visual inspection for turbidity after 7, 14, 21 and $28 \mathrm{~d}$ incubation.

\section{RESULTS AND DISCUSSION}

The 303 leptospire strains in this study represent 268 serovars in 30 named serogroups (Table 1). Fourteen strains were in new, thus far undesignated serogroups. As defined in a previous study (Brenner et al., 1993), the term genomospecies is used to indicate a species determined on the basis of genetic methods. Genomospecies corresponds to a DNA relatedness group. It is convenient to use genomospecies for unnamed species until they have been formally described. DNA hybridization comparisons divided the 303 strains studied into 17 DNA relatedness groups, each of which corresponds to the genetic designation of a species as 'strains with approximately $70 \%$ or greater DNA-DNA relatedness and with $5^{\circ} \mathrm{C}$ or less $\Delta T_{\mathrm{m}}$. Both values must be considered.' (Wayne et al., 1987). In our laboratory, we have long used DNA relatedness at a stringent reassociation temperature $\left(70^{\circ} \mathrm{C}\right.$ in the present study) as a third criterion for speciation, or for differentiating between species (Brenner, 1991). At the stringent criterion, strains of a single species are $60 \%$ or more related, whereas strains from different species exhibit significantly less relatedness as shown in our previous study (Yasuda et al., 1987) and in Tables 2 and 3 of the present study. The DNA relatedness results shown in Tables 2 and 3 illustrate the use of $70{ }^{\circ} \mathrm{C}$ relatedness values as a rapid screening method to either include or exclude strains from any given species. For example, a large number of strains exhibiting $69-100 \%$ relatedness in $70^{\circ} \mathrm{C}$ reactions to labelled DNA from $L$. interrogans serovar icterohaemorrhagiae were included in this species without testing for relatedness at $60^{\circ} \mathrm{C}$ or for divergence (Table 2). Similarly, relatedness values of less than $50 \%$ obtained at $70{ }^{\circ} \mathrm{C}$ were considered sufficient to differentiate between species. DNA relatedness results for 44 of the serovars included in this study were previously reported by Yasuda et al. (1987). Some of these were retested and others were not. Data from the Yasuda $e t$ al. (1987) study are indicated as explained in Table 2.

The largest number of strains (91 strains representing 82 different serovars) were in $L$. interrogans (Tables 1 and 2). Other species containing large numbers of strains were $L$. santarosai ( 65 strains from 59 serovars), $L$. borgpetersenii (49 strains from 43 serovars), $L$. 
Table 1. Strains of Leptospiraceae used in DNA relatedness studies

Blank spaces indicate that data are not available. Serovars given in bold type are not included in the list of Kmety \& Dikken (1988, 1993).

\begin{tabular}{|c|c|c|c|c|c|}
\hline Serovar & Serogroup & Strain & Species & Country of isolation & Source \\
\hline abrahamson & Hebdomadis & Abrahamson & L. santarosai & Panama & Human \\
\hline abramis & Pyrogenes & Abraham & L. interrogans & Malaysia & Human \\
\hline age & Undesignated & $\mathrm{AGC}$ & L. interrogans & Peru & Human \\
\hline agogo & Djasiman & Agogo & L. kirschneri & Ghana & Human \\
\hline aguaruna & Shermani & MW-4 & L. inadai & Peru & Opossum \\
\hline aguatia & Tarassovi & $45-74$ & L. santarosai & Peru & Cattle \\
\hline alexi & Pyrogenes & HS-616 & L. santarosai & Puerto Rico & Human \\
\hline alexi & Pyrogenes & Linaires & L. santarosai & Panama & Human \\
\hline alice & Autumnalis & Alice & L. santarosai & Sri Lanka & Human \\
\hline andamana & Andamana & $\mathrm{CH} 11$ & L. biflexa & Andaman Islands & Human \\
\hline anhoa & Celledoni & LT $90-68$ & L. borgpetersenii & Vietnam & Human \\
\hline arborea & Ballum & Arborea & L. borgpetersenii & Italy & Wood mouse \\
\hline argentiniensis & Bataviae & Peludo & L. noguchii & Argentina & Armadillo \\
\hline atchafalaya & Tarassovi & LSU 1013 & L. santarosai & USA & Opossum \\
\hline atlantae & Tarassovi & LT 81 & L. santarosai & USA & Opossum \\
\hline australis & Australis & Ballico & L. interrogans & Australia & Human \\
\hline autumnalis & Autumnalis & Akiyami A & L. interrogans & Japan & Human \\
\hline babudieri & Shermani & CI 40 & L. santarosai & Peru & Pig \\
\hline bac 1376 & Tarassovi & Bac 1376 & L. noguchii & Peru/Panama & Human \\
\hline bafani & Canicola & Bafani & L. kirschneri & Zaire & Human \\
\hline bagua & Pyrogenes & MW-12 & L. santarosai & Peru & Opossum \\
\hline bajan & Undesignated & Bajan & L. noguchii & Barbados & Toad \\
\hline bakeri & Tarassovi & LT 79 & L. santarosai & USA & Opossum \\
\hline balboa & Bataviae & $735 \mathrm{U}$ & L. santarosai & Panama & Spiny rat \\
\hline balcanica & Sejroe & 1627 Burgas & L. borgpetersenii & Bulgaria & Human \\
\hline balcanica & Sejroe & New Zealand & L. borgpetersenii & New Zealand & Opossum \\
\hline ballum & Ballum & Mus 127 & L. borgpetersenii & Denmark & Field mouse \\
\hline ballum & Ballum & $\mathrm{S} 102$ & L. borgpetersenii & Netherlands & \\
\hline bananal & Undesignated & Aa 14 & L. santarosai & Brazil & Field mouse \\
\hline bangkinang & Autumnalis & Bangkinang I & L. interrogans & Indonesia & Human \\
\hline bangkok & Australis & Bangkok D-92 & L. interrogans & Thailand & Dog \\
\hline barbudensis & Australis & Toad 67 & L. noguchii & Barbados & Toad \\
\hline bataviae & Bataviae & Van Tienen & L. interrogans & Indonesia & Human \\
\hline bataviae & Bataviae & Schoolby & L. santarosai & Panama & Human \\
\hline benjamini & Canicola & Benjamin & L. interrogans & Indonesia & Human \\
\hline beye & Mini & $1537 \mathrm{U}$ & L. santarosai & Panama & Spiny rat \\
\hline biflexa & Undesignated & 965 & Leptonema illini & USA & \\
\hline biflexa & Undesignated & LT 430 & L. inadai & USA & \\
\hline biggis & Pyrogenes & Biggs & L. interrogans & Malaysia & Human \\
\hline bim & Autumnalis & 1051 & L. kirschneri & Barbados & Dog \\
\hline bindjei & Canicola & Bindjei & L. interrogans & Indonesia & Human \\
\hline birkini & Icterohaemorrhagiae & Birkin & L. interrogans & Malaysia & Human \\
\hline bogvere & Icterohaemorrhagiae & LT $60-69$ & L. kirschneri & Jamaica & Rat \\
\hline borincana & Hebdomadis & HS 622 & L. santarosai & Puerto Rico & Human \\
\hline borincana & Hebdomadis & Norland & L. santarosai & Panama & Human \\
\hline borincana & Hebdomadis & Samson & L. santarosai & Panama & Human \\
\hline borincana & Hebdomadis & Woerner & L. santarosai & Panama & Human \\
\hline brasiliensis & Bataviae & An 776 & L. santarosai & Brazil & Opossum \\
\hline bratislava & Australis & Jez Bratislava & L. interrogans & Czechoslovakia & Hedgehog \\
\hline bravo & Tarassovi & Bravo & L. santarosai & Panama & Human \\
\hline broomi & Canicola & Patane & L. interrogans & Australia & Human \\
\hline budapest & Icterohaemorrhagiae & PV 1 & L. interrogans & Hungary & Laboratory rat \\
\hline bulgarica & Autumnalis & Mallika & L. interrogans & India & Human \\
\hline bulgarica & Autumnalis & Nicolaevo & L. kirschneri & Bulgaria & Human \\
\hline butembo & Autumnalis & Butembo & L. kirschneri & Zaire & Human \\
\hline camlo & Pyrogenes & LT 64-67 & L. interrogans & Vietnam & Human \\
\hline canalzonae & Grippotyphosa & CZ 188 & L. santarosai & Panama & Spiny rat \\
\hline canicola & Canicola & Hond Utrech IV & L. interrogans & Netherlands & Dog \\
\hline canicola & Canicola & Ruebush & L. interrogans & & \\
\hline caribe & Sejroe & TRVL 61866 & L. santarosai & Trinidad & Rat \\
\hline carimagua & Shermani & 9160 & L. noguchii & Argentina & \\
\hline carlos & Autumnalis & $\mathrm{C}-3$ & L. interrogans & Philippines & Toad \\
\hline castellonis & Ballum & Castellon 3 & L. borgpetersenii & Spain & Wood mouse \\
\hline celledoni & Celledoni & Celledoni & L. weilii & Australia & Human \\
\hline cenepa & Pyrogenes & $\mathrm{MW}-2$ & L. santarosai & Peru & Opossum \\
\hline ceylonica & Javanica & Piyasena & L. borgpetersenii & Sri Lanka & Human \\
\hline chagres & Tarassovi & $1913 \mathrm{~K}$ & L. santarosai & Panama & Spiny rat \\
\hline claytoni & Bataviae & $1348 \mathrm{U}$ & L. noguchii & Panama & Spiny rat \\
\hline codice & Codice & $\mathrm{CDC}$ & L. wolbachii & USA & \\
\hline copenhageni & Icterohaemorrhagiae & M 20 & L. interrogans & Denmark & Human \\
\hline copenhageni & Icterohaemorrhagiae & 'Virulent' & L. interrogans & & \\
\hline copenhageni & Icterohaemorrhagiae & Wijinberg & L. interrogans & Holland & Human \\
\hline
\end{tabular}


Table 1 (cont.)

\begin{tabular}{|c|c|c|c|c|c|}
\hline Serovar & Serogroup & Strain & Species & Country of isolation & Source \\
\hline cornelli & Pomona & $\mathrm{CB}$ & L. interrogans & USA & Cow \\
\hline $\operatorname{coxi}$ & Javanica & $\operatorname{Cox}$ & L. weilii & Malaysia & Human \\
\hline cristobali & Panama & LT 940 & L. noguchii & Panama & Opossum \\
\hline cynopteri & Cynopteri & $3522 \mathrm{C}$ & L. kirschneri & Indonesia & Bat \\
\hline dakota & Icterohaemorrhagiae & Grand River & L. kirschneri & USA & Water \\
\hline dania & Pomona & K 1 & L. santarosai & Denmark & Cow \\
\hline darien & Tarassovi & $637 \mathrm{~K}$ & L. santarosai & Panama & Opossum \\
\hline dehong & Javanica & De 10 & L. borgpetersenii & China & Suncus murinus \\
\hline dikkeni & Sejroe & Mannuthi & L. borgpetersenii & India & Bandicoot \\
\hline djasiman & Autumnalis & Djasiman & L. interrogans & Indonesia & Human \\
\hline djatzi & Bataviae & HS 26 & L. kirschneri & Puerto Rico & Human \\
\hline dukou & Canicola & 83194 & L. interrogans & China & \\
\hline erinaceiauriti & Autumnalis & Erineceus Auritus 670 & L. kirschneri & Russia & Hedgehog \\
\hline evansi & Ranarum & $267-1348$ & L. interrogans & Malaysia & Water \\
\hline figeiro & Hebdomadis & Figeiro & L. santarosai & Panama & Human \\
\hline fluminense & Javanica & Aa 3 & L. santarosai & Brazil & Field mouse \\
\hline fortbragg & Autumnalis & Fort Bragg & L. noguchii & USA & Human \\
\hline fugis & Australis & Fudge & L. interrogans & Malaysia & Human \\
\hline galtoni & Canicola & LT 1014 & L. kirschneri & Argentina & Cow \\
\hline gatuni & Tarassovi & $1473 \mathrm{~K}$ & L. santarosai & Panama & Opossum \\
\hline gem & Icterohaemorrhagiae & Simon & L. interrogans & Sri Lanka & Human \\
\hline gengma & Tarassovi & M 48 & L. borgpetersenii & China & Pig \\
\hline gent & Undesignated & Wa Gent & L. wolbachii & Belgium & Water \\
\hline georgia & Mini & LT 117 & L. santarosai & USA & Racoon \\
\hline geyaweera & Sejroe & Geyaweera & L. interrogans & Sri Lanka & Human \\
\hline goiano & Hebdomadis & Bovino 131 & L. santarosai & Brazil & Cow \\
\hline gorgas & Sejroe & $1413 \mathrm{U}$ & L. santarosai & Panama & Spiny rat \\
\hline grippotyphosa & Grippotyphosa & Andaman & L. interrogans & & \\
\hline grippotyphosa & Grippotyphosa & Moskva V & L. kirschneri & Russia & Human \\
\hline grippotyphosa & Grippotyphosa & $\mathrm{DF}$ & L. kirschneri & USA & Human \\
\hline grippotyphosa & Grippotyphosa & GG & L. kirschneri & USA & Human \\
\hline grippotyphosa & Grippotyphosa & STP & L. kirschneri & USA & Water \\
\hline guangdong (Ballum 3) & Ballum & 1853 & L. borgpetersenii & China & Rattus losea \\
\hline guaratuba & Pyrogenes & An 7705 & L. interrogans & Brazil & Opossum \\
\hline guaricura & Sejroe & Bov. G & L. santarosai & Brazil & Cow \\
\hline guidae & Tarassovi & RP 29 & L. horgpetersenii & Brazil & Pig \\
\hline gurungi & Djasiman & Gurung & L. interrogans & Malaysia & Human \\
\hline habaki & Bataviae & Habaki & Leptonema illini & & \\
\hline haemolytica & Sejroe & Marsh & L. interrogans & Malaysia & Human \\
\hline hainan & Celledoni & 6712 & L. weilii & China & Human \\
\hline hamptoni & Pyrogenes & Hampton & L. borgpetersenii & Malaysia & Human \\
\hline harbola & Javanica & Harbola 20 & L. borgpetersenii & & \\
\hline hardjo & Sejroe & $\mathrm{K}-125$ & L. borgpetersenii & USA & Cow \\
\hline hardjo & Sejroe & $\mathrm{T}-20$ & L. borgpetersenii & USA & Cow \\
\hline hardjo & Sejroe & Sponselee & L. borgpetersenii & Holland & Cow \\
\hline hardjo & Sejroe & Hardjoprajitno & L. interrogans & Indonesia & Human \\
\hline hardjo & Sejroe & Went 5 & L. meyeri & Canada & \\
\hline hawain & Australis & LT $62-68$ & L. interrogans & New Guinea & Bandicoot \\
\hline hebdomadis & Hebdomadis & Hebdomadis & L. interrogans & Japan & Human via Guinea pig \\
\hline hekou & Mini & H 27 & L. weilii & China & Human \\
\hline holland & Holland & WaZ Holland ${ }^{T}$ & Genomospecies 3 & Netherlands & Water \\
\hline honghe & Icterohaemorrhagiae & $\mathrm{H} 2^{\mathrm{T}}$ & L. interrogans & China & Human \\
\hline hualin & Icterohaemorrhagiae & LT $11-33$ & Genomospecies 4 & China & \\
\hline huallaga & Djasiman & M 7 & L. noguchii & Peru & Opossum \\
\hline icterohaemorrhagiae & Icterohaemorrhagiae & RGA & L. interrogans & Belgium & Human \\
\hline icterohaemorrhagiae & Icterohaemorrhagiae & 1 (Japan) & L. interrogans & Japan & Human \\
\hline icterohaemorrhagiae & Icterohaemorrhagiae & 1 (Kmety) & L. inadai & Japan & Human \\
\hline illini & Leptonema & 3055 & Leptonema illini & USA & Cow \\
\hline istrica & Hebdomadis & Bratislava & L. borgpetersenii & Czechoslovakia & Wood mouse \\
\hline jalna & Australis & Jalna & L. interrogans & Czechoslovakia & Yellow throat mouse \\
\hline javanica & Javanica & Veldrat Batavia 46 & L. borgpetersenii & Indonesia & Field rat \\
\hline jin & Sejroe & A81 & L. interrogans & China & Human \\
\hline jonsis & Canicola & Jones & L. interrogans & Malaysia & Human \\
\hline jules & Hebdomadis & Jules & L. borgpetersenii & Zaire & Human \\
\hline kabura & Hebdomadis & Kabura & L. kirschneri & Zaire & Human \\
\hline kambale & Hebdomadis & Kambale & L. kirschneri & Zaire & Human \\
\hline kamituga & Canicola & Kamituga & L. kirschneri & Zaire & Human \\
\hline kanana & Tarassovi & Kanana & L. borgpetersenii & Kenya & Gerbil \\
\hline kaup & Tarassovi & LT 64-68 & L. inadai & New Guinea & Bandicoot \\
\hline kennewicki & Pomona & LT 1026 & L. interrogans & USA & Cow \\
\hline kenya & Ballum & Nijenga & L. borgpetersenii & Kenya & Pouched rat \\
\hline kisuba & Tarassovi & Kisuba & L. borgpetersenii & Zaire & Human \\
\hline kobbe & Bataviae & $\mathrm{CZ} 320$ & L. santarosai & Panama & Spiny rat \\
\hline kremastos & Hebdomadis & Kremastos & L. interrogans & Australia & Human \\
\hline kremastos & Hebdomadis & $2414 \mathrm{VAB}$ & L. santarosai & Peru/Panama & Human \\
\hline kunming & Pomona & K 5 & L. kirschneri & China & Apodemus chevrieri \\
\hline kuwait & Canicola & $136 / 2 / 2$ & L. interrogans & Kuwait & Rat \\
\hline kwale & Pyrogenes & Julu & L. borgpetersenii & Kenya & Human \\
\hline
\end{tabular}


Table 1 (cont.)

\begin{tabular}{|c|c|c|c|c|c|}
\hline Serovar & Serogroup & Strain & Species & Country of isolation & Source \\
\hline lai & Icterohaemorrhagiae & Lai & L. interrogans & China & Human \\
\hline lambwe & Autumnalis & Lambwe & L. kirschneri & Kenya & Unstripped grass rat \\
\hline langati & Tarassovi & M 39039 & L. weilii & Malaysia & \\
\hline lanka & Louisiana & $\mathrm{R} / 740$ & L. interrogans & Sri Lanka & Human \\
\hline liangguang & Grippotyphosa & 1880 & L. interrogans & China & Rat \\
\hline lichuan (Manhao 4) & Manhao & Li 130 & L. inadai & China & Human \\
\hline lincang & Manhao & L 14 & L. inadai & China & Human \\
\hline longnan & Hebdomadis & L 573 & L. weilii & China & Human \\
\hline lora & Australis & Lora & L. interrogans & Italy & Human \\
\hline losbanos & Bataviae & LT $101-69$ & L. interrogans & Philippines & Rat \\
\hline louisiana & Louisiana & LSU 1945 & L. noguchii & USA & Armadillo \\
\hline luis & Shermani & M 6 & L. santarosai & Peru & Opossum \\
\hline lushui (Manhao 1) & Manhao & L 70 & Genomospecies 2 & China & \\
\hline lyme & Lyme & 10 & L. inadai & USA & Human \\
\hline machiguenga & Sarmin & MMD 3 & L. santarosai & Peru & Opossum \\
\hline malaya & Canicola & H 6 & L. inadai & Malaysia & Human \\
\hline mangus & Panama & TVRL/CAREC 137774 & L. inadai & Trinidad & Mongoose \\
\hline manhao 3 & Manhao & $\mathrm{L} 60^{\mathrm{T}}$ & Genomospecies 2 & China & \\
\hline manilae & Pyrogenes & LT 398 & L. interrogans & Philippines & Rat \\
\hline mankarso & Icterohaemorrhagiae & Mankarso & L. interrogans & Indonesia & Human \\
\hline manzhuang & Hebdomadis & A 23 & Genomospecies 2 & China & Human \\
\hline maru & Hebdomadis & CZ 285 & L. santarosai & Panama & Water via hamster \\
\hline maru & Hebdomadis & Brinkman & L. santarosai & Panama & Human \\
\hline maru & Hebdomadis & Clark & L. santarosai & Panama & Human \\
\hline may & Javanica & May & L. santarosai & Panama & Human \\
\hline medanensis & Sejroe & Hond $\mathrm{HC}$ & L. interrogans & Indonesia & Dog \\
\hline mengdeng & Celledoni & M 6906 & L. weilii & China & Human \\
\hline mengla & Javanica & A 85 & Genomospecies 2 & China & Human \\
\hline menglian & Pyrogenes & S 621 & L. weilii & China & Human \\
\hline mengma & Javanica & S 590 & L. weilii & China & Human \\
\hline menoni & Javanica & Kerala & L. borgpetersenii & Indonesia & Bandicoot \\
\hline menrun & Javanica & A 102 & L. weilii & China & Human \\
\hline $\operatorname{mini}$ & Mini & Sari & L. borgpetersenii & Italy & Human \\
\hline mogdeni & Tarassovi & Compton 746 & L. weilii & United Kingdom & Sewage \\
\hline moldaviae & Bataviae & $114-2$ & L. borgpetersenii & Russia & \\
\hline monjakov & Pomona & Monjakov & L. interrogans & Russia & Human \\
\hline monymusk & Icterohaemorrhagiae & LT 75-68 & L. interrogans & Jamaica & Rat \\
\hline monymusk & Icterohaemorrhagiae & 81552 & L. interrogans & China & \\
\hline mooris & Autumnalis & Moores & L. interrogans & Malaysia & Human \\
\hline mozdok & Pomona & 5621 & L. kirschneri & Russia & Field vole \\
\hline muenchen & Australis & München $\mathrm{C} 90$ & L. interrogans & Germany & Human \\
\hline mwogolo & Icterohaemorrhagiae & Mwogolo & L. kirschneri & Zaire & Human \\
\hline mwogolo & Icterohaemorrhagiae & Korea & L. interrogans & Korea & Human \\
\hline myocastoris & Pyrogenes & LSU 1551 & L. noguchii & USA & Nutria \\
\hline naam & Icterohaemorrhagiae & Naam & L. interrogans & Indonesia & Human \\
\hline nanding & Hebdomadis & M 6901 & Genomospecies 2 & China & Human \\
\hline nanxi & Icterohaemorrhagiae & HK 6 & L. interrogans & China & Human \\
\hline naparuca & Cynopteri & $\mathrm{NN}-1$ & L. santarosai & Peru & Galictis furax \\
\hline navet & Tarassovi & TRVL 109873 & L. santarosai & Trinidad & Human \\
\hline ndahambukuje & Icterohaemorrhagiae & Ndahambukuje & L. kirschneri & Zaire & Human \\
\hline ndambari & Icterohaemorrhagiae & Ndambari & L. kirschneri & Zaire & Human \\
\hline nero & Sejroe & Gamsulin & L. borgpetersenii & Russia & Human \\
\hline nicaragua & Australis & 1011 & L. noguchii & Nicaragua & Mustela nivalis \\
\hline nona & Hebdomadis & Nona & L. borgpetersenii & Zaire & Human \\
\hline nyanza & Sejroe & Kibos & L. borgpetersenii & Kenya & Human \\
\hline orleans & Louisiana & LSU 2580 & L. noguchii & USA & Nutria \\
\hline paidjan & Bataviae & Paidjan & L. interrogans & Indonesia & Human \\
\hline panama & Panama & $\mathrm{CZ} 214 \mathrm{~K}$ & L. noguchii & Panama & Opossum \\
\hline parva & Turneria & $\mathrm{H}$ & L. parva & England & Bacteriological media \\
\hline patoc & Semaranga & Patoc I & L. biflexa & Italy & Water \\
\hline perameles & Mini & Bandicoot 343 & L. meyeri & Australia & Perameles \\
\hline peru & Undesignated & MW 10 & L. santarosai & Peru & Opossum \\
\hline peruviana & Australis & V 42 & L. noguchii & Peru & Cow \\
\hline pina & Australis & LT 932 & L. borgpetersenii & Panama & Opossum \\
\hline pingchang & Ranarum & $80-412$ & Genomospecies 1 & China & Frog \\
\hline poi & Javanica & Poi & L. borgpetersenii & Italy & Human \\
\hline polonica & Sejroe & 493 Poland & L. borgpetersenii & Poland & Hedgehog \\
\hline pomona & Pomona & Pomona & L. interroguns & Australia & Human \\
\hline pomona & Pomona & 164 & L. interrogans & USA & Cow \\
\hline pomona & Pomona & S91 & L. interrogans & USA & Pig \\
\hline pomona & Pomona & Wickard & L. interrogans & USA & Cow \\
\hline pomona & Pomona & Johnson & L. interrogans & & \\
\hline pomona & Pomona & $24 \mathrm{~K}$ & L. noguchii & Russia & \\
\hline portlandvere & Canicola & My 1039 & L. interrogans & Jamaica & Human \\
\hline princestown & Pyrogenes & TRVL 112499 & L. santarosai & Trinidad & Human \\
\hline proechimys & Pomona & $1161 \mathrm{U}$ & L. noguchii & Panama & Spiny rat \\
\hline pyrogenes & Pyrogenes & Salinem & L. interrogans & Indonesia & Human \\
\hline pyrogenes & Pyrogenes & Northrup & L. santarosai & Panama & Human \\
\hline
\end{tabular}


Table 1 (cont.)

\begin{tabular}{|c|c|c|c|c|c|}
\hline Serovar & Serogroup & Strain & Species & Country of isolation & Source \\
\hline qingshui (Manhao 2) & Manhao & L 105 & L. weilii & China & Human \\
\hline qunjian & Canicola & 7957 & L. interrogans & China & Rat \\
\hline rachmati & Autumnalis & Rachmat & L. interrogans & Indonesia & Human \\
\hline rama & Tarassovi & 316 & L. santarosai & Panama & Opossum \\
\hline ramisi & Australis & Musa & L. kirschneri & Kenya & Human \\
\hline ranarum & Ranarum & ICF & L. meyeri & USA & Frog \\
\hline ranarum shu & Undesignated & Ranarum shu & L. interrogans & & \\
\hline ratnapura & Grippotyphosa & Wumalasena & L. kirschneri & Sri Lanka & Human \\
\hline recreo & Sejroe & 380 & L. interrogans & Nicaragua & Opossum \\
\hline ricardi & Sejroe & Richardson & L. interrogans & Malaysia & Human \\
\hline rio & Sarmin & $\operatorname{Rr} 5$ & L. santarosai & Brazil & Rat \\
\hline rioja & Bataviae & MR 12 & L. santarosai & Peru & Opossum \\
\hline robinsoni & Pyrogenes & Robinson & L. interrogans & Australia & Human \\
\hline roumanica & Sejroe & TM 294 & L. interrogans & Romania & Mus musculus \\
\hline ruparupae & Mini & M 3 & L. santarosai & Peru & Opossum \\
\hline rushan & Australis & 507 & L. noguchii & China & Bombina orientalis \\
\hline sanmartini & Pyrogenes & CT 63 & L. santarosai & Peru & Cow \\
\hline saopaulo & Semaranga & Sao Paulo ${ }^{T}$ & Genomospecies 5 & Brazil & Water \\
\hline sarmin & Sarmin & Sarmin & L. weilii & Indonesia & Human \\
\hline saxkoebing & Sejroe & Mus 24 & L. interrogans & Denmark & Wood mouse \\
\hline schueffneri & Canicola & Vleermuis & L. interrogans & Indonesia & Bat \\
\hline sejroe & Sejroe & M 84 & L. borgpetersenii & Denmark & Mouse \\
\hline semaranga & Semaranga & Veldrat Samarang & L. meyeri & Indonesia & Rat \\
\hline sentot & Djasiman & Sentot & L. interrogans & Indonesia & Human \\
\hline shermani & Shermani & $1342 \mathrm{~K}$ & L. santarosai & Panama & Spiny rat \\
\hline sichuan & Undesignated & $79601^{\mathrm{T}}$ & L. genomospecies 1 & China & Frog \\
\hline smithi & Icterohaemorrhagiae & Smith & L. interrogans & Malaysia & Human \\
\hline soccoestomes & Ballum & $78-082387$ & L. borgpetersenii & & \\
\hline sofia & Javanica & Sofia 874 & L. meyeri & Bulgaria & Human \\
\hline sorexjalna & Javanica & Sorex Jalna & L. borgpetersenii & Czechoslovakia & Shrew \\
\hline srebarna & Autumnalis & $1409 / 69$ & L. borgpetersenii & Bulgaria & Sorex auraneus \\
\hline sulzerae & Tarassovi & LT 82 & L. santarosai & USA & \\
\hline sumneri & Canicola & Sumner & L. interrogans & Malaysia & Human \\
\hline szwajizak & Mini & Szwajizak & L. interrogans & Australia & Human \\
\hline szwajizak & Mini & Oregon & L. santarosai & USA & Cow \\
\hline tabaquite & Mini & TVRL 3405 & L. santarosai & Trinidad & Human \\
\hline tarassovi & Tarassovi & Perepelicin & L. borgpetersenii & Russia & Human \\
\hline tingomariensis & Cynopteri & M 13 & L. santarosai & Peru & Opossum \\
\hline tonkini & Icterohaemorrhagiae & LT 96-68 & L. borgpetersenii & Vietnam & Human \\
\hline trinidad & Sejroe & TRVL 34056 & L. santarosai & Trinidad & Human \\
\hline tropica & Pomona & CZ 299 & L. santarosai & Panama & Spiny rat \\
\hline tsaratsovo & Pomona & B $81 / 7$ & L. kirschneri & Bulgaria & Harvest mouse \\
\hline tunis & Tarassovi & $\mathrm{P} 2 / 65$ & L. borgpetersenii & Tunisia & Pig \\
\hline Undesignated & Undesignated & 965 & Leptonema illini & USA & Unknown \\
\hline Undesignated & Undesignated & LT 430 & L. inadai & USA & Unknown \\
\hline unipertama & Sejroe & $\mathrm{K} 2-1$ & L. weilii & Indonesia & Cow \\
\hline valbuzzi & Grippotyphosa & Valbuzzi & L. interrogans & Australia & Human \\
\hline valbuzzi & Grippotyphosa & Dyster & L. kirschneri & & \\
\hline vanderhoedeni & Grippotyphosa & Kipod 179 & L. kirschneri & Israel & Hedgehog \\
\hline varela & Pyrogenes & 1019 & L. santarosai & Nicaragua & Opossum \\
\hline vargonicas & Javanica & 24 & L. santarosai & Peru & Rodent \\
\hline vughia & Tarassovi & LT 89-68 & L. weilii & Vietnam & Human \\
\hline waskurin & Sarmin & LT $63-68$ & L. interrogans & New Guinea & Bandicoot \\
\hline wawain & Undesignated & MW 6 & L. santarosai & Peru & Opossum \\
\hline weaveri & Sarmin & $\mathrm{CZ} 390$ & L. santarosai & Panama & Human \\
\hline weerasinghe & Autumnalis & Weerasinghe & L. interrogans & Sri Lanka & Human \\
\hline wewak & Australis & LT $65-68$ & L. interrogans & New Guinea & Dog \\
\hline whitcombi & Celledoni & Whitcomb & L. borgpetersenii & Malaysia & Human \\
\hline wolffi & Sejroe & 3705 & L. interrogans & Indonesia & Human \\
\hline worsfoldi & Hebdomadis & Worsfold & L. borgpetersenii & Malaysia & Human \\
\hline yaan & Javanica & $80-27$ & L. borgpetersenii & China & Crocidura attenuata \\
\hline yunnan & Mini & A 10 & Genomospecies 2 & China & Human \\
\hline yunxian & Tarassovi & L 100 & L. borgpetersenii & China & Pig \\
\hline zanoni & Pyrogenes & Zanoni & L. interrogans & Australia & Human \\
\hline zhenkang & Javanica & L 82 & L. borgpetersenii & China & Rat \\
\hline K 128 & Undesignated & K 128 & L. borgpetersenii & & \\
\hline K 142 & Undesignated & K 142 & L. borgpetersenii & & \\
\hline$\times \mathbf{4 7}$ & Sejroe & $\times 47$ & L. santarosai & Indonesia & \\
\hline $21-74$ & Bataviae & $21-74$ & L. interrogans & Brazil & \\
\hline $26-73$ & Bataviae & 3859 & L. interrogans & Indonesia & \\
\hline $27-75$ & Javanica & Azalia & L. inadai & Indonesia & Human \\
\hline $52-73$ & Javanica & 457 & L. borgpetersenii & Sri Lanka & Dog \\
\hline 82224 & Icterohaemorrhagiae & 82224 & L. interrogans & China & \\
\hline $83-011457$ & Tarassovi & MOIK & L. santarosai & Panama & \\
\hline 83-015437 & Undesignated & W16K & L. noguchii & Panama & \\
\hline $84-011370$ & Undesignated & 2050 & L. noguchii & Panama & Human \\
\hline $87-029496$ & Hebdomadis & KF001 & L. santarosai & Panama & Human \\
\hline
\end{tabular}


Table 2. DNA relatedness of strains within Leptospira and Leptonema species

$\mathrm{RBR}$, Relative binding ratio $=[($ percentage DNA bound to hydroxyapatite in heterologous reactions $) /($ percentage DNA bound in homologous reactions) $] \times 100$. D, Percentage divergence (calculated to the nearest $0.5 \%$ ); calculations of $\mathrm{D}$ assumed that a $1 \%$ decrease in the thermal stability of a heterologous DNA duplex compared with that of the homologous duplex was caused by $1 \%$ of the bases within the duplex that were unpaired. Values shown in bold type were taken from our previous study (Yasuda et al., 1987). For example, in the reaction between serovar icterohaemorrhagiae RGA and serovar djasiman, the $55^{\circ} \mathrm{C}$ value was obtained from the first study and the $70^{\circ} \mathrm{C}$ value was obtained in the present study. For serovars where more than one strain was studied, the strain names are given in parentheses.

\begin{tabular}{|c|c|c|c|}
\hline Unlabelled DNA from serovar & $\mathrm{RBR}$ at $55^{\circ} \mathrm{C}$ & D & RBR at $70^{\circ} \mathrm{C}$ \\
\hline & \multicolumn{3}{|c|}{ Labelled DNA from $L$. interrogans serovar icterohaemorrhagiae strain RGA } \\
\hline icterohaemorrhagiae (RGA) & 100 & $0 \cdot 0$ & 100 \\
\hline agc & $100^{*}$ & & $100^{*}$ \\
\hline australis & $100^{*}$ & & $100^{*}$ \\
\hline autumnalis & $100^{*}$ & & $100^{*}$ \\
\hline djasiman & $100^{*}$ & & 100 \\
\hline jalna & $100^{*}$ & & 100 \\
\hline pyrogenes (Salinem) & $100^{*}$ & & $100^{*}$ \\
\hline schueffneri & $100^{*}$ & & 100 \\
\hline lanka & & & 100 \\
\hline mooris & & & 100 \\
\hline pomona (Pomona) & 100 & 1.5 & 100 \\
\hline saxkoebing & 100 & 1.0 & \\
\hline bataviae (Van Tienen) & $100^{*}$ & & 99 \\
\hline grippotyphosa (Andaman) & $100^{*}$ & $0.0^{*}$ & 99* \\
\hline zanoni & 100 & & 90 \\
\hline 82224 & 100 & $3 \cdot 0$ & 86 \\
\hline smithi & 100 & & 77 \\
\hline guaratuba & $100 \dagger$ & $2.5 \dagger$ & $69 \dagger$ \\
\hline bulgarica (Mallika) & 99 & $1 \cdot 0$ & 97 \\
\hline broomi & 99 & $1 \cdot 0$ & 93 \\
\hline wolffi & 98 & 0.0 & 100 \\
\hline muenchen & 98 & $1 \cdot 5$ & 85 \\
\hline honghe & 97 & 0.5 & 87 \\
\hline robinsoni & 95 & $2 \cdot 0$ & 73 \\
\hline mwogolo (Korea) & & & 95 \\
\hline losbanos & 94 & $1 \cdot 0$ & 89 \\
\hline copenhageni (Wijinberg) & 94 & 0.5 & 89 \\
\hline $21-74$ & & & 94 \\
\hline fugis & & & 94 \\
\hline canicola (Hond Utrech IV) & 93 & $\mathbf{1 . 0}$ & 93 \\
\hline qunjian & 93 & $1 \cdot 5$ & 78 \\
\hline huwain & 93 & $4 \cdot 5$ & 77 \\
\hline roumanica & & & 93 \\
\hline $26-73$ & & & 93 \\
\hline copenhageni (M20) & 92 & 0.0 & 100 \\
\hline sumneri & 92 & 0.5 & 93 \\
\hline abramis & & & 92 \\
\hline sentot & & & 92 \\
\hline copenhageni ('virulent') & 91 & $1 \cdot 0$ & 100 \\
\hline liangguang & 91 & 0.5 & 92 \\
\hline jin & 91 & $1 \cdot 5$ & 87 \\
\hline nanxi & 91 & $2 \cdot 0$ & 87 \\
\hline gurungi & & & 91 \\
\hline monjakov & & & 91 \\
\hline monymusk (LT 75-68) & & & 91 \\
\hline manilae & & & 91 \\
\hline ricardi & & & 91 \\
\hline
\end{tabular}


Table 2 (cont.)

\begin{tabular}{|c|c|c|c|}
\hline Unlabelled DNA from serovar & $\mathrm{RBR}$ at $55^{\circ} \mathrm{C}$ & $\mathbf{D}$ & RBR at $70^{\circ} \mathrm{C}$ \\
\hline & \multicolumn{3}{|c|}{ Labelled DNA from $L$. interrogans serovar icterohaemorrhagiae strain RGA } \\
\hline biggis & 90 & 0.5 & 96 \\
\hline birkini & 90 & 0.5 & 96 \\
\hline kennewicki & 90 & $1 \cdot 0$ & 89 \\
\hline mankarso & 90 & 0.5 & 78 \\
\hline carlos & 90 & 1.5 & 75 \\
\hline bratislava & & & 90 \\
\hline icterohaemorrhagiae 1 (Japan) & 89 & 0.5 & 91 \\
\hline hebdomadis & 89* & 3.0* & 91 \\
\hline rachmati & & & 89 \\
\hline cornelli & 88 & 0.5 & 94 \\
\hline jonsis & 88 & $2 \cdot 5$ & 83 \\
\hline dukou & 88 & 1.0 & 79 \\
\hline bindjei & & & 88 \\
\hline bangkok & & & 87 \\
\hline pomona (164) & & & 87 \\
\hline lai & 86 & 1.5 & 91 \\
\hline benjamini & & & 86 \\
\hline haemolytica & 85 & 1.0 & 94 \\
\hline medanensis & & & 85 \\
\hline valbuzzi (Valbuzzi) & & & 85 \\
\hline weerasinghe & & & 85 \\
\hline monymusk (81552) & 84 & $2 \cdot 0$ & 96 \\
\hline ranarum shu & 84 & 1.5 & 89 \\
\hline canicola (Ruebush) & & & 84 \\
\hline hardjo (Hardjoprajitno) & & & 84 \\
\hline geyaweera & & & 83 \\
\hline waskurin & 82 & $3 \cdot 5$ & 69 \\
\hline pomona (Johnson) & & & 82 \\
\hline wewak & 81 & 3.5 & 65 \\
\hline bangkinang & & & 81 \\
\hline pomona (S 91) & & & 81 \\
\hline camlo & & & 80 \\
\hline evansi & & & 80 \\
\hline portlandvere & & & 80 \\
\hline gem & & & 79 \\
\hline kremastos (Kremastos) & & & 79 \\
\hline naam & & & 79 \\
\hline pomona (Wickard) & & & 79 \\
\hline lora & & & 78 \\
\hline recreo & & & 77 \\
\hline szwajizak (Szwajizak) & & & 76 \\
\hline budapest & & & 74 \\
\hline paidjan & & & 72 \\
\hline \multirow[t]{2}{*}{ kuwait } & & & 69 \\
\hline & \multicolumn{3}{|c|}{ Labelled DNA from $L$. santarosai serovar shermani strain $1342 \mathrm{~K}$} \\
\hline shermani & 100 & 0.0 & 100 \\
\hline sulzerae & $100 \dagger$ & & \\
\hline alexi & & & 100 \\
\hline gorgas & & & 100 \\
\hline kobbe & & & 100 \\
\hline princestown & & & 100 \\
\hline ruparupae & & & 100 \\
\hline sanmartini & & & 100 \\
\hline
\end{tabular}


Table 2 (cont.)

\begin{tabular}{|c|c|c|c|}
\hline Unlabelled DNA from serovar & $\mathrm{RBR}$ at $55^{\circ} \mathrm{C}$ & $\mathbf{D}$ & RBR at $70^{\circ} \mathrm{C}$ \\
\hline & \multicolumn{3}{|c|}{ Labelled DNA from $L$. santarosai serovar shermani strain $1342 \mathrm{~K}$} \\
\hline tropica & & & 100 \\
\hline varela & & & 100 \\
\hline cenepa & 97 & $1 \cdot 5$ & 95 \\
\hline bravo & 97 & $3 \cdot 5$ & \\
\hline balboa & 96 & $1 \cdot 5$ & 86 \\
\hline trinidad & & & 96 \\
\hline figeiro & 95 & $1 \cdot 5$ & 84 \\
\hline atlantae & 93 & 0.5 & 87 \\
\hline bakeri & 92 & 2.0 & $\mathbf{8 8}$ \\
\hline bagua & & & 92 \\
\hline tingomaria & & & 91 \\
\hline borincana & 90 & 0.5 & 91 \\
\hline alice & 90 & 1.5 & 79 \\
\hline beye & & & 90 \\
\hline chagres & & & 88 \\
\hline guaricura & & & 88 \\
\hline machiguenga & & & 88 \\
\hline peru & 86 & 0.5 & 91 \\
\hline bananal & 86 & 0.5 & 90 \\
\hline wawain & 86 & 0.0 & 86 \\
\hline navet & 86 & $2 \cdot 0$ & 86 \\
\hline$\times 47$ & & & 86 \\
\hline maru & & & 85 \\
\hline pyrogenes (Northrup) & 85 & $2 \cdot 0$ & 77 \\
\hline naparuca & & & 84 \\
\hline canalzonae & & & 83 \\
\hline 83-011457 & & & 83 \\
\hline atchafalaya & 82 & $2 \cdot 5$ & 99 \\
\hline borincana (Woerner) & 82 & $3 \cdot 0$ & 79 \\
\hline abrahamson & 82 & 1.0 & 72 \\
\hline weaveri & & & 82 \\
\hline babudieri & & & 81 \\
\hline darien & & & 81 \\
\hline rama & & & 81 \\
\hline tabaquite & & & 81 \\
\hline borincana (Sampson) & 80 & 3.0 & 79 \\
\hline aquatia & & & 80 \\
\hline gatuni & & & 80 \\
\hline riojo & 79 & 1.0 & 87 \\
\hline borincana (Norland) & 79 & $3 \cdot 0$ & 76 \\
\hline goiano & 78 & $1 \cdot 0$ & \\
\hline kremastos (2414 VAB) & 78 & $3 \cdot 0$ & 68 \\
\hline may & 78 & $2 \cdot 0$ & 66 \\
\hline maru (Clark) & 78 & $2 \cdot 5$ & 66 \\
\hline luis & & & 78 \\
\hline rio & & & 78 \\
\hline bataviae (Schoolby) & 76 & 1.5 & 69 \\
\hline alexi (Linaires) & 74 & $1 \cdot 0$ & \\
\hline dania & 74 & $1 \cdot 5$ & 69 \\
\hline caribe & & & 74 \\
\hline vargonicus & & & 74 \\
\hline szwajizak (Oregon) & 71 & $1 \cdot 0$ & 53 \\
\hline fluminense & & & 71 \\
\hline
\end{tabular}


Table 2 (cont.)

\begin{tabular}{|c|c|c|c|}
\hline Unlabelled DNA from serovar & RBR at $55^{\circ} \mathrm{C}$ & $\mathbf{D}$ & RBR at $70^{\circ} \mathrm{C}$ \\
\hline & \multicolumn{3}{|c|}{ Labelled DNA from $L$. santarosai serovar shermani strain $1342 \mathrm{~K}$} \\
\hline georgia & & & 71 \\
\hline maru (Brinkman) & 70 & $1 \cdot 5$ & 58 \\
\hline $87-029496$ & 69 & $1 \cdot 0$ & 72 \\
\hline \multirow[t]{2}{*}{ brasiliensis } & & & 68 \\
\hline & \multicolumn{3}{|c|}{ Labelled DNA from $L$. borgpetersenii serovar javanica strain Veldrat Batavia 46} \\
\hline javanica (Veldrat Batavia 46) & 100 & $0 \cdot 0$ & 100 \\
\hline K 142 & 100 & $1 \cdot 0$ & 95 \\
\hline soccoestomes & & & 100 \\
\hline yaan & & & 100 \\
\hline zhenkang & 98 & $1 \cdot 0$ & 89 \\
\hline dehong & 98 & 0.5 & 88 \\
\hline sorexjalna & & & 98 \\
\hline $\operatorname{mini}$ & 97 & & 80 \\
\hline kenya & 96 & 0.5 & 100 \\
\hline kanana & 96 & 0.5 & 92 \\
\hline castellonis & & & 96 \\
\hline ceylonica & & & 96 \\
\hline poi & 95 & $2 \cdot 0$ & 94 \\
\hline K 148 & 95 & 0.5 & 92 \\
\hline hardjo (K125) & 95 & $1 \cdot 0$ & 80 \\
\hline ballum (Mus 127) & 94 & $0 \cdot 0$ & 99 \\
\hline menoni & 94 & $1 \cdot 5$ & \\
\hline arborea & & & 94 \\
\hline dikkeni & 93 & 1.0 & 100 \\
\hline nyanza & 93 & $1 \cdot 0$ & 96 \\
\hline nana & 92 & 0.5 & 100 \\
\hline sejroe & 92 & $4 \cdot 5$ & 90 \\
\hline pina & 91 & $1 \cdot 0$ & 85 \\
\hline tarassovi & 90 & $1 \cdot 0$ & 75 \\
\hline nero & 89 & $1 \cdot 5$ & 93 \\
\hline guangdong & 88 & $1 \cdot 0$ & 86 \\
\hline harbala & $88 \ddagger$ & $1 \cdot 0 \ddagger$ & \\
\hline ballum (S 102) & 87 & $4 \cdot 5$ & 92 \\
\hline balcanica (New Zealand) & 87 & $1 \cdot 5$ & 74 \\
\hline jules & 86 & $1 \cdot 5$ & 98 \\
\hline hardjo (T 20) & 86 & $2 \cdot 0$ & 85 \\
\hline guidae & 86 & $2 \cdot 5$ & 76 \\
\hline balcanica (1627 Burgas) & & & 86 \\
\hline polonica & & & 86 \\
\hline yunxian & 85 & $2 \cdot 0$ & 98 \\
\hline moldaviae & 85 & $1 \cdot 5$ & 93 \\
\hline $52-73$ & 85 & $1 \cdot 0$ & 85 \\
\hline istrica & & & 85 \\
\hline kwale & & & 85 \\
\hline srebarna & & & 84 \\
\hline hardjo (Sponselee) & 83 & $2 \cdot 0$ & 82 \\
\hline anhoa & 82 & $2 \cdot 0$ & 82 \\
\hline tonkini & $80 \S$ & $2 \cdot 5 \S$ & \\
\hline kisuba & 78 & $1 \cdot 5$ & 74 \\
\hline gengma & 77 & $2 \cdot 0$ & 89 \\
\hline tunis & & & 77 \\
\hline worsfoldi & & & 74 \\
\hline hamptoni & $72 \|$ & $1 \cdot 5 \|$ & $67 \|$ \\
\hline whitcombi & & & 64 \\
\hline
\end{tabular}


Table 2 (cont.)

\begin{tabular}{|c|c|c|c|}
\hline Unlabelled DNA from serovar & $\mathrm{RBR}$ at $55^{\circ} \mathrm{C}$ & $\mathbf{D}$ & RBR at $70^{\circ} \mathrm{C}$ \\
\hline & \multicolumn{3}{|c|}{ Labelled DNA from L. kirschneri serovar cynopteri strain 3522C } \\
\hline cynopteri & & 0.0 & 100 \\
\hline grippotyphosa (DF) & \multirow[t]{2}{*}{100} & \multirow[t]{2}{*}{$2 \cdot 0$} & 97 \\
\hline ndahambukuje & & & 1009 \\
\hline tsaratsova & 98 & $1 \cdot 0$ & 94 \\
\hline kunming & 96 & $1 \cdot 0$ & 100 \\
\hline dakota & \multirow[t]{2}{*}{96} & \multirow[t]{2}{*}{$1 \cdot 0$} & 94 \\
\hline butembo & & & 969 \\
\hline bafani & 95 & 1.0 & 92 \\
\hline ratnapura & 94 & 2.5 & 81 \\
\hline bogvere & 93 & 1.0 & 84 \\
\hline grippotyphosa (GG) & \multirow[t]{3}{*}{92} & \multirow[t]{3}{*}{$2 \cdot 0$} & \\
\hline mozdok & & & $92 \uparrow$ \\
\hline ndambari & & & 90 \\
\hline kabura & 89 & $2 \cdot 0$ & 98 \\
\hline grippotyphosa (STP) & \multirow[t]{6}{*}{89} & \multirow[t]{6}{*}{$2 \cdot 0$} & 95 \\
\hline erinaceiauriti & & & 899 \\
\hline djatzi & & & $88 \pi$ \\
\hline mwogolo (Mwogolo) & & & 889 \\
\hline vanderhoedeni & & & 88 ฯ \\
\hline galtoni & & & $87 \%$ \\
\hline agogo & 86 & $2 \cdot 0$ & 91 \\
\hline kambale & 86 & $1 \cdot 0$ & 89 \\
\hline valbuzzi (Dyster) & \multirow[t]{2}{*}{86} & \multirow[t]{2}{*}{$1 \cdot 5$} & 83 \\
\hline bim & & & 869 \\
\hline bulgarica (Nicolaevo) & 85 & $2 \cdot 0$ & 80 \\
\hline lambwe & \multirow[t]{4}{*}{84} & \multirow[t]{4}{*}{$0 \cdot 0$} & \\
\hline grippotyphosa (Moskva V) & & & 839 \\
\hline kamituga & & & 829 \\
\hline \multirow[t]{2}{*}{ ramisi } & & & 739 \\
\hline & \multicolumn{3}{|c|}{ Labelled DNA from $L$. noguchii serovar panama strain CZ $214 \mathrm{~K}$} \\
\hline panama & 100 & $0 \cdot 0$ & 100 \\
\hline proechimys & 100 & & 89 \\
\hline pomona $(24 K)$ & 100 & 0.5 & 84 \\
\hline rushon & 99 & $1 \cdot 0$ & 78 \\
\hline carimagua & 95 & 1.5 & 87 \\
\hline huallaga & & & 92 \\
\hline barbudensis & 90 & $2 \cdot 5$ & 87 \\
\hline nicaragua & 90 & $3 \cdot 5$ & 66 \\
\hline orleans & 90 & 3.5 & 62 \\
\hline $83-015437$ & 89 & 1.5 & 68 \\
\hline bac 1376 & 87 & $2 \cdot 0$ & 93 \\
\hline fortbragg & 86 & $2 \cdot 5$ & 77 \\
\hline bajan & 85 & $2 \cdot 5$ & 79 \\
\hline louisiana & 84 & $2 \cdot 0$ & 77 \\
\hline claytoni & & & 84 \\
\hline myocastoris & 83 & $3 \cdot 5$ & 67 \\
\hline $84-011370$ & 81 & $3 \cdot 5$ & 71 \\
\hline argentiniensis & & & 78 \\
\hline cristobali (LT 960) & & & 76 \\
\hline peruviana & & & 70 \\
\hline
\end{tabular}


Table 2 (cont.)

\begin{tabular}{|c|c|c|c|}
\hline Unlabelled DNA from serovar & RBR at $55^{\circ} \mathrm{C}$ & $\mathbf{D}$ & RBR at $70^{\circ} \mathrm{C}$ \\
\hline & \multicolumn{3}{|c|}{ Labelled DNA from $L$. weilii serovar celledoni strain Celledoni } \\
\hline celledoni & 100 & $0 \cdot 0$ & 100 \\
\hline mengma & 100 & $0 \cdot 0$ & 96 \\
\hline vughia & \multirow[t]{3}{*}{100} & \multirow[t]{3}{*}{$2 \cdot 5$} & 82 \\
\hline hainan & & & 98 \\
\hline menrun & & & 98 \\
\hline hekou & 90 & $1 \cdot 0$ & 77 \\
\hline menglian & \multirow[t]{2}{*}{89} & \multirow[t]{2}{*}{$1 \cdot 5$} & 84 \\
\hline mogdeni & & & 89 \\
\hline sarmin & \multirow[t]{2}{*}{88} & \multirow[t]{2}{*}{$2 \cdot 0$} & 80 \\
\hline $\operatorname{coxi}$ & & & 87 \\
\hline mengdeng & 86 & $1 \cdot 0$ & 93 \\
\hline longnan & 86 & $1 \cdot 0$ & 83 \\
\hline unipertama & 80 & $2 \cdot 5$ & 82 \\
\hline langati & & & 77 \\
\hline \multirow[t]{2}{*}{ qingshui } & & & 72 \\
\hline & \multicolumn{3}{|c|}{ Labelled DNA from $L$, inadai serovar lyme strain 10} \\
\hline lyme & 100 & 0.0 & 100 \\
\hline $27-75$ & 100 & $0 \cdot 0$ & 97 \\
\hline lichuan & 100 & 1.5 & 94 \\
\hline lincang & 100 & $2 \cdot 0$ & 90 \\
\hline mangus & 99 & $0 \cdot 5$ & 100 \\
\hline malaya & 99 & $0 \cdot 0$ & 88 \\
\hline kaup & 98 & $0 \cdot 0$ & 96 \\
\hline aguarina & 98 & $0 \cdot 0$ & 91 \\
\hline icterohaemorrhagiae 1 (Kmety) & 89 & $0 \cdot 0$ & 98 \\
\hline \multirow[t]{2}{*}{ undesignated (LT 430) } & 78 & $0 \cdot 0$ & 81 \\
\hline & \multicolumn{3}{|c|}{ Labelled DNA from $L$. meyeri serovar ranarum strain ICF } \\
\hline ranarum & 100 & $0 \cdot 0$ & 100 \\
\hline sofia & 100 & $0 \cdot 0$ & 100 \\
\hline perameles & 100 & & \\
\hline hardjo (Went 5) & 94 & 0.5 & 91 \\
\hline \multirow[t]{2}{*}{ semaranga } & 83 & $2 \cdot 5$ & 86 \\
\hline & \multicolumn{3}{|c|}{ Labelled DNA from Leptonema illini serovar illini strain 3055} \\
\hline illini & 100 & $0 \cdot 0$ & 100 \\
\hline habaki & 100 & $2 \cdot 0$ & 75 \\
\hline \multirow[t]{2}{*}{ undesignated (965) } & 83 & $3 \cdot 0$ & 61 \\
\hline & \multicolumn{3}{|c|}{ Labelled DNA from $L$. wolbachii serovar codice strain CDC } \\
\hline codice & 100 & $0 \cdot 0$ & 100 \\
\hline \multirow[t]{2}{*}{ gent } & 100 & $2 \cdot 0$ & 93 \\
\hline & \multicolumn{3}{|c|}{ Labelled DNA from $L$. biflexa serovar andaman strain CH 11} \\
\hline andaman & 100 & $0 \cdot 0$ & 100 \\
\hline \multirow[t]{2}{*}{ patoc } & 98 & $1 \cdot 0$ & 74 \\
\hline & \multicolumn{3}{|c|}{ Labelled DNA from $L$. parva serovar parva strain $H$} \\
\hline \multirow[t]{2}{*}{ parva } & 100 & $0 \cdot 0$ & 100 \\
\hline & \multicolumn{3}{|c|}{ Labelled DNA from Leptospira genomospecies 1 serovar pingchang strain 80-41 } \\
\hline pingchang & 100 & 0.0 & 100 \\
\hline sichuan & $98 \dagger$ & & \\
\hline
\end{tabular}


Table 2 (cont.)

\begin{tabular}{|c|c|c|c|}
\hline Unlabelled DNA from serovar & RBR at $55^{\circ} \mathrm{C}$ & D & $\mathbf{R B R}$ at $70^{\circ} \mathrm{C}$ \\
\hline & \multicolumn{3}{|c|}{ Labelled DNA from Leptospira genomospecies 2 serovar manhao strain $\mathrm{L} 60^{\mathrm{T}}$} \\
\hline manhao 3 & 100 & $0 \cdot 0$ & 100 \\
\hline lushui & 86 & $1 \cdot 0$ & 90 \\
\hline mengla & 85 & 0.5 & 95 \\
\hline manzhuang & 85 & $0 \cdot 5$ & 85 \\
\hline yunnan & \multicolumn{3}{|c|}{ Labelled DNA from Leptospira genomospecies 3 serovar holland strain WaZ Holland } \\
\hline \multirow[t]{2}{*}{ holland } & 100 & $0 \cdot 0$ & 100 \\
\hline & \multicolumn{3}{|c|}{ Labelled DNA from Leptospira genomospecies 4 serovar hualin strain LT 11-33 } \\
\hline \multirow[t]{2}{*}{ hualin } & 100 & $0 \cdot 0$ & 100 \\
\hline & \multicolumn{3}{|c|}{ Labelled DNA from Leptospira genomospecies 5 serovar saopaulo strain Sao Paulo ${ }^{\mathrm{T}}$} \\
\hline
\end{tabular}

* Values were obtained using labelled L. interrogans serovar copenhageni M 20 DNA.

† Values obtained were with labelled DNA from the strain listed as unlabelled. For example, the values for serovar guaratuba were obtained using labelled DNA from serovar guaratuba and unlabelled DNA from serovar icterohaemorrhagiae strain RGA.

$¥$ Results obtained using labelled DNA from serovar Menoni.

$\S$ Results obtained using labelled DNA from serovar Anhoa.

\| Results obtained using labelled DNA from serovar Tonkini.

T Results obtained using labelled DNA from serovar Ndambari.

kirschneri (29 strains from 26 serovars) and L. noguchii (20 strains from 20 serovars). The remaining 49 strains each represented a different serovar. Those that belonged to named species were: $L$. weilii, 15 strains; $L$. inadai, 10 strains, L. meyeri, five strains; Leptonema illini, three strains; L. biflexa, two strains, $L$. wolbachii, two strains; and $L$. parva, one strain. Eleven strains belonged to previously unidentified species, which we designated Leptospira genomospecies 1-5. Two strains were in Leptospira genomospecies 1, six strains were in Leptospira genomospecies 2 and one strain was in each of Leptospira genomospecies 3, 4 and 5.

L. parva and Leptospira genomospecies 3, 4 and 5 contained a single strain that showed less than specieslevel relatedness to the type strain of all other species. The remaining named species, as well as Leptospira genomospecies 1 and 2, contained two or more strains (see exceptions below) that fulfil the molecular definition of a species: $70 \%$ relatedness at optimal DNA renaturation temperature $\left(55^{\circ} \mathrm{C}\right.$ in these studies) and whose related sequences exhibit $5 \%$ or less divergence [on the assumption that each $1{ }^{\circ} \mathrm{C}$ of decreased thermal stability of a heterologous DNA duplex compared with that of the homologous DNA duplex is caused by approximately $1 \%$ unpaired bases (Wayne et al., 1987)]. In our laboratory, we included the additional parameter that strains of the same species exhibit $60 \%$ or greater relatedness at a stringent DNA renaturation temperature $\left(70^{\circ} \mathrm{C}\right.$ in this study). DNA relatedness determined at the $70^{\circ} \mathrm{C}$ temperature was used as the sole criterion for including
112 of the 303 strains in a given species. Partial or complete data from our previous study (Yasuda et al., 1987) was used for 31 strains, as indicated by the bold numbers in Table 1 . Of the 299 strains included in species containing two or more strains, all but four strains showed relatedness and divergence values within the limits of the species definition. The exceptions were: strain 87-029496, which was $69 \%$ related to the type strain of $L$. santarosai at $55^{\circ} \mathrm{C}$ with $1.0 \%$ divergence and was $72 \%$ related to it at $70{ }^{\circ} \mathrm{C}$; strain Oregon of serovar szwajizak, which was $71 \%$ related to the type strain of $L$. santarosai at $55^{\circ} \mathrm{C}$ with $1.0 \%$ divergence and was $53 \%$ related to it at $70{ }^{\circ} \mathrm{C}$; strain 6901 of serovar nanding, which was $69 \%$ related to the type strain of Leptospira genomospecies 2 at $55^{\circ} \mathrm{C}$ with $1.0 \%$ divergence and was $81 \%$ related to it at $70{ }^{\circ} \mathrm{C}$; and strain A-10 of serovar yunnan, which was $66 \%$ related to the type strain of Leptospira genomospecies 2 at $55^{\circ} \mathrm{C}$ with $1.5 \%$ divergence and was $60 \%$ related to it at $70{ }^{\circ} \mathrm{C}$.

By definition, the type strain of every species must exhibit less than species-level relatedness to the type strain (and any other strain tested) of every other species. DNA relatedness ranges between species are shown in Table 3. In these comparisons, labelled DNAs from type and reference strains of each named species and genomospecies were reacted with unlabelled DNAs from the same and different species (see Table 3). Relatedness between a number of species pairs is close, but in all cases, the levels of relatedness observed within strains of a species are substantially 
Table 3. DNA relatedness between leptospire species

Strains used as sources of labelled DNA are as follows: L. interrogans, RGA; L. santarosai, 1342K; L. borgpetersenii, Veldrat Batavia 46; L. kirschneri, 3522C; L. noguchii, CZ 214 K; L. weilii, Celledoni; L. inadai, 10; L. meyeri, ICF; Leptonema illini, 3055; L. wolbachii, CDC; L. biflexa, CH 11; L. parva, H; Leptospira genomospecies 1, 80-412; Leptospira genomospecies 2 , L $60^{\mathrm{T}}$; Leptospira genomospecies 3, WaZ Holland ${ }^{\mathrm{T}}$; Leptospira genomospecies 4, LT 11-33; Leptospira genomospecies 5, Sao Paulo $^{\mathrm{T}} . n$, No. of strains studied.

\begin{tabular}{|c|c|c|c|c|c|c|c|c|c|c|c|c|c|c|c|c|c|c|}
\hline \multirow[t]{2}{*}{ Source of labelled DNA } & \multicolumn{18}{|c|}{ Relatedness $(\%)$ to unlabelled DNAs from: } \\
\hline & $55^{\circ} \mathrm{C}$ & $(n)$ & $\mathbf{D}$ & $(n)$ & $70^{\circ} \mathrm{C}$ & $(n)$ & $55^{\circ} \mathrm{C}$ & $(n)$ & $\mathbf{D}$ & $(n)$ & $70^{\circ} \mathrm{C}$ & $(n)$ & $55^{\circ} \mathrm{C}$ & $(n)$ & $\mathbf{D}$ & $(n)$ & $70^{\circ} \mathrm{C}$ & $(n)$ \\
\hline & \multicolumn{6}{|c|}{ L. interrogans } & \multicolumn{6}{|c|}{ L. santarosai } & \multicolumn{6}{|c|}{ L. borgpetersenii } \\
\hline L. interrogans & 93 & $(48)$ & $1 \cdot 0$ & (38) & 88 & (90) & 46 & $(10)$ & $10 \cdot 0$ & $(5)$ & 7 & $(66)$ & 51 & (4) & $11 \cdot 0$ & (1) & 6 & (43) \\
\hline L. santarosai & 34 & (3) & $12 \cdot 5$ & (1) & 6 & (4) & 84 & (30) & $1 \cdot 5$ & (29) & 82 & $(60)$ & 62 & (2) & $8 \cdot 0$ & (2) & 18 & (10) \\
\hline L. borgpetersenii & 33 & $(10)$ & 13.5 & (5) & 13 & (7) & 53 & (24) & $10 \cdot 0$ & (23) & 30 & (63) & 89 & (33) & 1.5 & (33) & 88 & (45) \\
\hline L. kirschneri & 39 & (1) & $12 \cdot 0$ & (2) & 30 & (4) & 50 & (1) & & & 9 & (6) & 50 & (1) & & & 9 & (6) \\
\hline L. noguchii & 66 & (8) & $9 \cdot 5$ & (4) & 41 & (9) & 48 & (8) & $14 \cdot 0$ & (4) & 9 & $(24)$ & 44 & (3) & $10 \cdot 5$ & (1) & 9 & (6) \\
\hline L. weilii & 43 & (2) & $14 \cdot 0$ & (1) & 8 & (6) & 57 & (9) & $9 \cdot 5$ & (9) & 24 & (26) & 71 & (2) & 6.5 & (2) & 28 & (3) \\
\hline L. inadai & 3 & (2) & & & 2 & (2) & 5 & (1) & & & 1 & (2) & 10 & (2) & & & 5 & (1) \\
\hline L. meyeri & 2 & (1) & & & 3 & (2) & 1 & (1) & & & 1 & (2) & 5 & (2) & & & 1 & (5) \\
\hline Leptonema illini & & & & & 0 & (2) & & & & & 0 & (2) & 5 & (1) & & & 0 & (6) \\
\hline L. wolbachii & 3 & (7) & & & 0 & (2) & 6 & (3) & & & 1 & (2) & 3 & (3) & & & 0 & (5) \\
\hline L. biftexa & 3 & (2) & & & 1 & (2) & 5 & (1) & & & 1 & (2) & 6 & (1) & & & 1 & (5) \\
\hline L. parva & 0 & (1) & & & 0 & (2) & 0 & (2) & & & 0 & (2) & 3 & (2) & & & 0 & (6) \\
\hline Leptospira genomospecies 1 & 35 & (1) & & & 6 & (2) & 42 & (2) & & & 12 & (3) & 44 & (4) & & & 13 & (4) \\
\hline Leptospira genomospecies 2 & & & & & 2 & (2) & & & & & 14 & (2) & 61 & (5) & 4.0 & (5) & 28 & (5) \\
\hline Leptospira genomospecies 3 & 5 & (1) & & & 1 & (1) & 2 & (1) & & & 0 & (1) & 5 & (2) & & & 1 & (2) \\
\hline Leptospira genomospecies 4 & 4 & (1) & & & 5 & (1) & 5 & (3) & & & 2 & (1) & 4 & (3) & & & 2 & (1) \\
\hline \multirow[t]{2}{*}{ Leptospira genomospecies 5} & 3 & (1) & & & & & 3 & (2) & & & & & 2 & (3) & & & & \\
\hline & \multicolumn{6}{|c|}{ L. kirschneri } & \multicolumn{6}{|c|}{ L. noguchii } & \multicolumn{6}{|c|}{ L. weilï } \\
\hline L. interrogans & 67 & $(21)$ & $7 \cdot 5$ & $(20)$ & 41 & (27) & 70 & (6) & $8 \cdot 0$ & (6) & 34 & $(18)$ & 38 & (1) & & & 7 & (15) \\
\hline L. santarosai & 23 & (2) & $15 \cdot 5$ & (1) & 6 & (24) & 41 & (1) & $13 \cdot 0$ & (1) & 8 & (10) & 54 & (2) & $10 \cdot 5$ & (2) & 25 & (9) \\
\hline L. borgpetersenii & 26 & (1) & $13 \cdot 5$ & (1) & 7 & (28) & 34 & (5) & 11.5 & (5) & 11 & (18) & 68 & (8) & 7.5 & (7) & 42 & (15) \\
\hline L. kirschneri & 92 & (15) & 1.5 & (15) & 89 & (26) & & & & & 23 & (4) & & & & & 5 & (2) \\
\hline L. noguchii & 66 & (14) & $9 \cdot 0$ & (14) & 31 & (24) & 90 & (14) & $2 \cdot 0$ & $(14)$ & 78 & (19) & 50 & (2) & 13.5 & (1) & 8 & (7) \\
\hline L. weilii & 47 & (1) & $11 \cdot 5$ & (1) & 7 & (14) & 50 & (1) & $13 \cdot 5$ & (1) & 5 & (8) & 90 & (8) & 1.5 & (8) & 86 & (14) \\
\hline L. inadai & 5 & (1) & & & 0 & (3) & 5 & (1) & & & 0 & (3) & 7 & (1) & & & 0 & (1) \\
\hline L. meyeri & 1 & (1) & & & 1 & (3) & & & & & 1 & (3) & 4 & (1) & & & 1 & (1) \\
\hline Leptonema illini & & & & & 0 & (4) & & & & & 0 & (3) & & & & & 0 & (1) \\
\hline L. wolbachii & & & & & 0 & (3) & 2 & (3) & & & 0 & (3) & 4 & (1) & & & 0 & (1) \\
\hline L. biflexa & 3 & (1) & & & 1 & (3) & 11 & (1) & & & 1 & (3) & 5 & (1) & & & 3 & (1) \\
\hline L. parva & 0 & (1) & & & 0 & (4) & 0 & (1) & & & 0 & (3) & & & & & 0 & (1) \\
\hline Leptospira genomospecies 1 & 33 & (1) & & & 2 & (2) & 21 & (1) & & & 4 & (3) & 43 & (1) & & & 3 & (1) \\
\hline Leptospira genomospecies 2 & & & & & 2 & (3) & & & & & 1 & (4) & 41 & (1) & 60 & (1) & 16 & (1) \\
\hline Leptospira genomospecies 3 & 5 & (1) & & & 1 & (1) & 4 & (1) & & & 1 & (3) & 5 & (1) & & & 0 & (1) \\
\hline Leptospira genomospecies 4 & 4 & (1) & & & 1 & (1) & 4 & (1) & & & 1 & (3) & 4 & (1) & & & & \\
\hline \multirow[t]{2}{*}{ Leptospira genomospecies 5} & 2 & (1) & & & & & 2 & (1) & & & & & 1 & (1) & & & & \\
\hline & \multicolumn{6}{|c|}{ L. inadai } & \multicolumn{6}{|c|}{ L. meyeri } & & & ptoner & allini & & \\
\hline L. interrogans & 5 & (1) & & & 2 & $(10)$ & 4 & (3) & & & 2 & (2) & 7 & (1) & & & 8 & (3) \\
\hline L. santarosai & 5 & (1) & & & 2 & $(10)$ & 3 & (1) & & & 3 & (5) & & & & & 5 & (3) \\
\hline L. borgpetersenii & 6 & (1) & & & 3 & (10) & 4 & (2) & & & 6 & (4) & 4 & (1) & & & 4 & (2) \\
\hline L. kirschneri & & & & & 3 & (10) & & & & & 1 & (2) & & & & & 4 & (3) \\
\hline L. noguchii & 7 & (1) & & & 3 & (10) & 3 & (2) & & & 3 & (4) & 6 & (1) & & & 2 & (2) \\
\hline L. weilii & & & & & 3 & (7) & 5 & (3) & & & 5 & (4) & 14 & (1) & & & 5 & (1) \\
\hline L. inadai & 96 & (9) & 0.5 & (9) & 93 & (9) & 3 & (3) & & & 4 & (2) & 6 & (1) & & & 0 & (3) \\
\hline L. meyeri & 1 & (1) & & & 1 & (10) & 94 & (4) & $1 \cdot 0$ & (3) & 92 & (3) & 3 & (1) & & & 1 & (3) \\
\hline Leptonema illini & & & & & 0 & (1) & & & & & 0 & (2) & 92 & (2) & 1.5 & (2) & 68 & (2) \\
\hline L. wolbachii & & & & & 1 & (1) & 41 & (3) & $11 \cdot 5$ & (1) & 14 & (2) & 2 & (1) & & & 1 & (2) \\
\hline L. biflexa & 3 & (1) & & & 1 & (9) & 30 & (2) & & & 3 & (3) & 0 & (1) & & & 1 & (3) \\
\hline L. parva & 0 & (1) & & & 0 & (1) & 1 & (3) & & & 2 & (1) & 2 & (1) & & & 0 & (3) \\
\hline Leptospira genomospecies 1 & 6 & (1) & & & 0 & (1) & 5 & (3) & & & 0 & (1) & 1 & (1) & & & 0 & (1) \\
\hline Leptospira genomospecies 2 & & & & & 0 & (1) & & & & & 1 & (2) & & & & & 10 & (1) \\
\hline Leptospira genomospecies 3 & 5 & (1) & & & 1 & (1) & 57 & (2) & $11 \cdot 0$ & (2) & 28 & (2) & 4 & (1) & & & 0 & (1) \\
\hline Leptospira genomospecies 4 & 4 & (1) & & & 0 & (1) & 54 & (2) & & & 14 & (1) & 2 & (1) & & & 1 & (1) \\
\hline Leptospira genomospecies 5 & 1 & (1) & & & & & 29 & (2) & & & & & 2 & (1) & & & & \\
\hline
\end{tabular}

higher than those observed between species. In no case do strains not included in a given species fulfil more than one of the three criteria used here for the molecular definition of a species $(70 \%$ or greater relatedness at $55^{\circ} \mathrm{C}, 5 \%$ or less divergence within related sequences and $60 \%$ or greater relatedness at $70{ }^{\circ} \mathrm{C}$ ). Examples of closely related species are $L$. interrogans and $L$. noguchii, L. borgpetersenii and 
Table 3 (cont.)

\begin{tabular}{|c|c|c|c|c|c|c|c|c|c|c|c|c|c|c|c|c|c|c|}
\hline \multirow[t]{2}{*}{ Source of labelled DNA } & \multicolumn{18}{|c|}{ Relatedness (\%) to unlabelled DNAs from: } \\
\hline & $55^{\circ} \mathrm{C}$ & $(n)$ & $\mathbf{D}$ & $(n)$ & $70^{\circ} \mathrm{C}$ & $(n)$ & $55^{\circ} \mathrm{C}$ & $(n)$ & D & (n) & $70^{\circ} \mathrm{C}$ & $(n)$ & $55^{\circ} \mathrm{C}$ & (n) & $\mathbf{D}$ & $(n)$ & $70^{\circ} \mathrm{C}$ & (n) \\
\hline & \multicolumn{6}{|c|}{ L. wolbachii } & \multicolumn{6}{|c|}{ L. biffexa } & \multicolumn{6}{|c|}{ L. parva } \\
\hline L. interrogans & 7 & (1) & & & 2 & (2) & 27 & (1) & & & 9 & (2) & 0 & (1) & & & 2 & (1) \\
\hline L. santarosai & & & & & 2 & (2) & & & & & 3 & (2) & 1 & (1) & & & & \\
\hline L. borgpetersenii & & & & & 6 & (2) & 9 & (2) & & & 0 & (1) & 2 & (1) & & & 1 & (1) \\
\hline L. kirschneri & & & & & 2 & (1) & & & & & 1 & (2) & & & & & 1 & (1) \\
\hline L. noguchii & 5 & (1) & & & 5 & (2) & 21 & (2) & & & 3 & (2) & 1 & (1) & & & 3 & (1) \\
\hline L. weillii & & & & & 7 & (2) & 13 & (2) & & & 6 & (2) & 3 & (1) & & & 7 & (1) \\
\hline L. inadai & 11 & (1) & & & 1 & (2) & 4 & (1) & & & 4 & (1) & 7 & (1) & & & 0 & (1) \\
\hline L. meyeri & 25 & (1) & & & 16 & (2) & & & & & 4 & (1) & & & & & 1 & (1) \\
\hline Leptonema illini & & & & & 0 & (1) & & & & & 0 & (1) & & & & & 0 & (1) \\
\hline L. wolbachii & 100 & (1) & $2 \cdot 0$ & (1) & 92 & (1) & 15 & (2) & & & 6 & (1) & & & & & 1 & (1) \\
\hline L. biflexa & 21 & (1) & & & 13 & (1) & 98 & (1) & 1.0 & (1) & 74 & (1) & 0 & (1) & & & 2 & (1) \\
\hline L. parva & 1 & (1) & & & 0 & (1) & 0 & (1) & & & 0 & (1) & & & & & & \\
\hline Leptospira genomospecies 1 & 2 & (1) & & & 0 & (1) & & & & & 0 & (1) & 2 & (1) & & & 0 & (1) \\
\hline Leptospira genomospecies 2 & & & & & 1 & (1) & & & & & 0 & (1) & & & & & 0 & (1) \\
\hline Leptospira genomospecies 3 & 74 & (1) & 6.0 & (1) & 45 & (1) & 41 & (1) & & & 6 & (1) & 8 & (1) & & & 0 & (1) \\
\hline Leptospira genomospecies 4 & 58 & (1) & $10 \cdot 5$ & (1) & 20 & (1) & 39 & (1) & & & 5 & (1) & 2 & (1) & & & 1 & (1) \\
\hline \multirow[t]{2}{*}{ Leptospira genomospecies 5} & 26 & (1) & & & 41 & (1) & & & & & & & 1 & (1) & & & & \\
\hline & \multicolumn{6}{|c|}{ Leptospira genomospecies 1} & \multicolumn{6}{|c|}{ Leptospira genomospecies 2} & \multicolumn{6}{|c|}{ Leptospira genomospecies 3} \\
\hline L. interrogans & & & & & 7 & (1) & & & & & 5 & (5) & & & & & 4 & (1) \\
\hline L. santarosai & & & & & 6 & (1) & 66 & (1) & & & 18 & (6) & & & & & 1 & (1) \\
\hline L. borgpetersenii & & & & & 25 & (1) & 70 & (5) & 6.5 & (5) & 45 & (6) & & & & & 1 & (1) \\
\hline L. kirschneri & & & & & 7 & (1) & & & & & 7 & (5) & & & & & 1 & (1) \\
\hline L. noguchii & & & & & 5 & (1) & & & & & 5 & (5) & & & & & 2 & (1) \\
\hline L. weilii & & & & & 18 & (1) & & & & & 42 & (5) & & & & & 2 & (1) \\
\hline L. inadai & & & & & 1 & (1) & & & & & 1 & (4) & & & & & 1 & (1) \\
\hline L. meyeri & & & & & 0 & (1) & & & & & 1 & (5) & & & & & 12 & (l) \\
\hline Leptonema illini & & & & & 0 & (1) & & & & & 1 & (5) & & & & & 0 & (1) \\
\hline L. wolbachii & & & & & 1 & (1) & & & & & 0 & (5) & 60 & (1) & 6.5 & (1) & 48 & (1) \\
\hline L. biffexa & & & & & 0 & (1) & & & & & 1 & (5) & & & & & 6 & (1) \\
\hline L. parva & & & & & 1 & (1) & & & & & 1 & (6) & & & & & 0 & (1) \\
\hline Leptospira genomospecies 1 & 98 & (1) & & & & & 40 & (1) & & & 16 & (1) & 3 & (1) & & & & \\
\hline Leptospira genomospecies 2 & & & & & 11 & (1) & 79 & (5) & $1 \cdot 0$ & (5) & 82 & (5) & & & & & 2 & (1) \\
\hline Leptospira genomospecies 3 & 9 & (1) & & & 0 & (1) & 4 & (1) & & & & & & & & & & \\
\hline Leptospira genomospecies 4 & 2 & (1) & & & & & 4 & (1) & & & & & 55 & (1) & & & 25 & (1) \\
\hline \multirow[t]{2}{*}{ Leptospira genomospecies 5} & 2 & (2) & & & & & 1 & (1) & & & & & 26 & (1) & & & & \\
\hline & \multicolumn{6}{|c|}{ Leptospira genomospecies 4} & \multicolumn{6}{|c|}{ Leptospira genomospecies 5} & & & & & & \\
\hline L. interrogans & & & & & 7 & (1) & & & & & 3 & (1) & & & & & & \\
\hline L. santarosai & & & & & 1 & (1) & & & & & 1 & (1) & & & & & & \\
\hline L. borgpetersenii & & & & & 1 & (1) & & & & & 2 & (1) & & & & & & \\
\hline L. kirschneri & & & & & 1 & (1) & & & & & & & & & & & & \\
\hline L. noguchii & & & & & 2 & (1) & & & & & 2 & (1) & & & & & & \\
\hline L. weilii & & & & & 6 & (1) & & & & & 4 & (1) & & & & & & \\
\hline L. inadai & & & & & 1 & (1) & & & & & 3 & (1) & & & & & & \\
\hline L. meyeri & & & & & 14 & (1) & & & & & 7 & (1) & & & & & & \\
\hline Leptonema illini & & & & & 0 & (1) & & & & & 0 & (1) & & & & & & \\
\hline L. wolbachii & 10 & (1) & 10.5 & (1) & 21 & (1) & & & & & 8 & (1) & & & & & & \\
\hline L. biflexa & & & & & 5 & (1) & & & & & & & & & & & & \\
\hline L. parva & & & & & 0 & (1) & & & & & 0 & (1) & & & & & & \\
\hline Leptospira genomospecies 1 & 4 & (1) & & & 0 & (1) & & & & & & & & & & & & \\
\hline Leptospira genomospecies 2 & & & & & 1 & (1) & & & & & 1 & (1) & & & & & & \\
\hline Leptospira genomospecies 3 & 63 & (1) & $13 \cdot 5$ & (1) & 20 & (1) & & & & & & & & & & & & \\
\hline Leptospira genomospecies 4 & & & & & & & & & & & & & & & & & & \\
\hline Leptospira genomospecies 5 & 28 & (1) & & & & & & & & & & & & & & & & \\
\hline
\end{tabular}

Leptospira genomospecies 2, $L$. weilii and $L$. borgpetersenii, and L. wolbachii and Leptospira genomospecies 3 .

The results of this study confirmed and extended our previous finding (Yasuda et al., 1987) and that of Ramadass et al. (1992) of species heterogeneity among serovars of a given serogroup. Of a total of 24 serogroups, in which two or more serovars were tested, only the seven strains in serogroup Ballum were confined to a single species (Table 4). All other serogroups in which more than one serovar were tested were identified in from two to six species. Similarly, all species containing more than one serovar contained serovars belonging to two to seventeen serogroups (Table 4). Species heterogeneity was also found when multiple strains of a single serovar were tested (Table 5). Two to six strains from each of 20 serovars were tested. In only eight of these did all strains belong to the same species. It is therefore not possible to determine the species of any serogroup or serovar without specifically identifying the strain. 
Table 4. Distribution of serogroups among leptospire species

Species: 1, L. interrogans; 2, L. santarosai; 3, L. borgpetersenii; 4, L. kirschneri; 5, L. noguchii; 6, L. weilii; 7, L. inadai; 8, L. meyeri; 9, Leptonema illini; 10, L. wolbachii; 11, L. biflexa; 12, L. parva; 13, Genomospecies 1; 14, Genomospecies $2 ; 15$, Genomospecies 3; 16, Genomospecies 4; 17, Genomospecies 5.

\begin{tabular}{|c|c|c|c|c|c|c|c|c|c|c|c|c|c|c|c|c|c|c|}
\hline \multirow[t]{2}{*}{ Serogroup } & \multirow{2}{*}{$\begin{array}{l}\text { No. of } \\
\text { strains }\end{array}$} & \multicolumn{17}{|c|}{ No. of strains in species } \\
\hline & & 1 & 2 & 3 & 4 & 5 & 6 & 7 & 8 & 9 & 10 & 11 & 12 & 13 & 14 & 15 & 16 & 17 \\
\hline Andamana & 1 & & & & & & & & & & & 1 & & & & & & \\
\hline Australis & 15 & 9 & & 1 & 1 & 4 & & & & & & & & & & & & \\
\hline Autumnalis & 16 & 8 & 1 & 1 & 5 & 1 & & & & & & & & & & & & \\
\hline Ballum & 7 & & & 7 & & & & & & & & & & & & & & \\
\hline Bataviae & 15 & 5 & 5 & 1 & 1 & 2 & & & & 1 & & & & & & & & \\
\hline Canicola & 16 & 12 & & & 3 & & & 1 & & & & & & & & & & \\
\hline Celledoni & 5 & & & 2 & & & 3 & & & & & & & & & & & \\
\hline Codice & 1 & & & & & & & & & & 1 & & & & & & & \\
\hline Cynopteri & 3 & & 2 & & 1 & & & & & & & & & & & & & \\
\hline Djasiman & 4 & 2 & & & 1 & 1 & & & & & & & & & & & & \\
\hline Grippotyphosa & 11 & 3 & 1 & & 7 & & & & & & & & & & & & & \\
\hline Hebdomadis & 23 & 2 & 12 & 4 & 2 & & 1 & & & & & & & & 2 & & & \\
\hline Icterohaemorrhagiae & 26 & 18 & & 1 & & 5 & & 1 & & & & & & & & & 1 & \\
\hline Javanica & 19 & & 3 & 10 & & & 3 & 1 & 1 & & & & & & 1 & & & \\
\hline Leptonema & 1 & & & & & & & & & 1 & & & & & & & & \\
\hline Louisiana & 3 & 1 & & & & 2 & & & & & & & & & & & & \\
\hline Lyme & 1 & & & & & & & 1 & & & & & & & & & & \\
\hline Manhao & 5 & & & & & & 1 & 2 & & & & & & & 2 & & & \\
\hline Mini & 10 & 1 & 5 & 1 & & & 1 & & 1 & & & & & & 1 & & & \\
\hline Panama & 3 & & & & & 2 & & 1 & & & & & & & & & & \\
\hline Pomona & 15 & 8 & 2 & & 3 & 2 & & & & & & & & & & & & \\
\hline Pyrogenes & 20 & 8 & 8 & 2 & & 1 & 1 & & & & & & & & & & & \\
\hline Ranarum & 3 & 1 & & & & & & & 1 & & & & & 1 & & & & \\
\hline Sarmin & 5 & 1 & 3 & & & & 1 & & & & & & & & & & & \\
\hline Sejroe & 27 & 10 & 5 & 10 & & & 1 & & 1 & & & & & & & & & \\
\hline Semaranga & 3 & & & & & & & & 1 & & 1 & & & & & & & 1 \\
\hline Shermani & 5 & & 3 & & & 1 & & 1 & & & & & & & & & & \\
\hline Tarassovi & 24 & & 12 & 7 & & 1 & 3 & 1 & & & & & & & & & & \\
\hline Turneria & 1 & & & & & & & & & & & & 1 & & & & & \\
\hline Undesignated & 15 & 2 & 3 & 2 & & 3 & & 1 & & 1 & 1 & & & 1 & & 1 & & \\
\hline
\end{tabular}

Our previous study indicated that the biochemical tests used to differentiate $L$. interrogans sensu lato from $L$. biflexa sensu lato were of no value in differentiating the then 11 species of Leptospiraceae (Yasuda et al., 1987). This remains true for $L$. kirschneri, described by Ramadass et al. (1992), and for the five new Leptospira genomospecies identified in the present study. Results of phenotypic characterization of 119 strains are presented in Table 6 . Only one of six $L$. inadai strains grew at $11{ }^{\circ} \mathrm{C}$ and only four of the 119 strains grew well at $37^{\circ} \mathrm{C}$. Although all leptospires grow slowly, all strains grew well at $30^{\circ} \mathrm{C}$. Strains of $L$. inadai, $L$. biflex $a$ and $L$. wolbachii were variable for growth in the presence of the inhibitory compound 8-azaguanine. $L$. meyeri (two strains tested) and single strains of $L$. parva, Leptonema illini and Leptospira genomospecies 4 and 5 grew in the presence of 8-azaguanine; strains of all other species did not grow. Only L. meyeri (two strains) grew in the presence of the inhibitor 2,6- diaminopurine. Strains of $L$. interrogans, $L$. santarosai, $L$. borgpetersenii, $L$. noguchii, $L$. weilii, $L$. inadai and $L$. biflexa gave variable results for growth in 2,6diaminopurine; strains of the remaining species did not grow. Growth in the presence of copper sulfate was positive in two L. meyeri strains and in single strains of L. parva, Leptospira genomospecies 4 and Leptospira genomospecies 5; growth was negative in strains of $L$. interrogans, $L$. santarosai, $L$. borgpetersenii, $L$. noguchii, Leptonema illini, Leptospira genomospecies 1 and Leptospira genomospecies 3 and variable in strains of the other species. The presence of lipase activity was variable among species; strains of $L$. interrogans, $L$. noguchii, L. inadai, L. kirschneri and Leptospira genomospecies 2 were variable, although largely positive. The small number of strains tested in $L$. biflexa, $L$. meyeri, L. wolbachii, L. parva and Leptonema illini all possessed lipase activity, whereas strains of $L$. borgpetersenii, L. santarosai, L. weilii and Leptospira 
Table 5. Species distribution of serovars in which multiple strains were tested

Figures in parentheses indicate the number of strains in each species.

\begin{tabular}{|lcl|}
\hline Serovar & $\begin{array}{c}\text { No. strains } \\
\text { tested }\end{array}$ & \multicolumn{1}{c|}{ Species to which serovars belong } \\
\hline alexi & 2 & L. santarosai $(2)$ \\
balcanica & 2 & L. borgpetersenii $(2)$ \\
ballum & 2 & L. borgpetersenii $(2)$ \\
bataviae & 2 & L. interrogans $(1)$, L. santarosai $(1)$ \\
borincana & 4 & L. santarosai $(4)$ \\
bulgarica & 2 & L. interrogans $(1)$, L. kirschneri $(1)$ \\
canicola & 2 & L. interrogans $(2)$ \\
copenhageni & 3 & L. interrogans $(3)$ \\
grippotyphosa & 5 & L. kirschneri $(4)$, L. interrogans $(1)$ \\
hardjo & 5 & L. borgpetersenii $(3)$, L. interrogans $(1)$, L. meyeri $(1)$ \\
icterohaemorrhagiae & 3 & L. interrogans $(2)$, L. inadai $(1)$ \\
kremastos & 2 & L. interrogans $(1)$, L. santarosai $(1)$ \\
maru & 3 & L. santarosai $(3)$ \\
monymusk & 2 & L. interrogans $(2)$ \\
mwogolo & 2 & L. kirschneri $(1)$, L. interrogans $(1)$ \\
pomona & 6 & L. interrogans $(5)$, L. noguchii $(1)$ \\
pyrogenes & 2 & L. interrogans $(1)$, L. santarosai $(1)$ \\
szwajizak & 2 & L. interrogans $(1)$, L. santarosai $(1)$ \\
valbuzzi & 2 & L. interrogans $(1)$, L. kirschneri $(1)$ \\
\hline
\end{tabular}

\section{Table 6. Phenotypic characteristics of leptospires}

8-AG, 8-Azaguanine; 2,6-DAP, 2,6-diaminopurine; - , $10 \%$ or fewer strains positive;,$+ 90 \%$ or more strains positive; $\mathrm{v}, 10-89 \%$ strains positive.

\begin{tabular}{|c|c|c|c|c|c|c|c|c|}
\hline \multirow[t]{2}{*}{ Species } & \multirow[t]{2}{*}{$\begin{array}{l}\text { No. of } \\
\text { strains }\end{array}$} & \multicolumn{3}{|c|}{$\begin{array}{c}\text { Growth at } \\
\text { temp. }\left({ }^{\circ} \mathrm{C}\right) \text { of : }\end{array}$} & \multicolumn{3}{|c|}{$\begin{array}{l}\text { Growth in the } \\
\text { presence of: }\end{array}$} & \multirow[t]{2}{*}{$\begin{array}{l}\text { Lipase } \\
\text { activity }\end{array}$} \\
\hline & & 11 & 30 & 37 & 8-AG & 2,6-DAP & $\begin{array}{l}\text { Copper } \\
\text { sulfate }\end{array}$ & \\
\hline L. interrogans & 28 & - & + & - & - & $\mathrm{v}$ & - & $\mathrm{v}$ \\
\hline L. santarosai & 28 & - & + & - & - & $\mathrm{v}$ & - & - \\
\hline L. borgpetersenii & 16 & - & + & - & - & $\mathrm{v}$ & - & - \\
\hline L. noguchii & 8 & - & + & - & - & $\mathrm{v}$ & - & $\mathbf{v}$ \\
\hline L. weilii & 6 & - & + & - & - & $\mathrm{V}$ & $\mathrm{v}$ & - \\
\hline L. inadai & 6 & $\mathbf{v}$ & + & $\mathrm{v}$ & $\mathrm{v}$ & $\mathrm{v}$ & $\mathrm{v}$ & $\mathrm{v}$ \\
\hline L. kirschneri & 6 & - & + & - & - & - & $\mathrm{v}$ & $\mathrm{v}$ \\
\hline L. biflexa & 3 & - & + & $\mathrm{v}$ & $\mathrm{v}$ & $\mathrm{v}$ & $\mathrm{v}$ & + \\
\hline L. meyeri & 2 & - & + & + & + & + & + & + \\
\hline L. wolbachii & 2 & - & + & - & $\mathrm{v}$ & - & $\mathrm{v}$ & + \\
\hline Leptonema illini & 2 & - & + & $\mathrm{v}$ & + & - & - & + \\
\hline L. parva & 1 & - & + & + & + & - & + & + \\
\hline Genomospecies 1 & 2 & - & + & - & - & - & - & - \\
\hline Genomospecies 2 & 5 & - & + & - & - & - & $\mathrm{v}$ & $\mathrm{v}$ \\
\hline Genomospecies 3 & 1 & - & + & - & - & - & - & - \\
\hline Genomospecies 4 & 1 & - & + & - & + & - & + & - \\
\hline Genomospecies 5 & 1 & - & + & - & + & - & + & - \\
\hline
\end{tabular}

genomospecies 1, 3, 4 and 5 lacked lipase activity. These reactions are presently of little or no value in differentiating species of Leptospiraceae.
Our findings, although quite preliminary, suggest that the geographic distribution of species is not totally random. There are eight species in which we have 
identified six or more strains (Table 1). The six strains of Leptospira genomospecies 2 were all isolated in China. All but three of the 65 strains of $L$. santarosai were from North and South America, as were all but two of the 20 strains of $L$. noguchii. Nearly half of the $91 \mathrm{~L}$. interrogans strains were isolated from countries in Oceania, representing more than $70 \%$ of the total isolates from this area. Additional study is needed to determine whether other factors are responsible for this restricted distribution. Thus far, all 37 African Leptospira strains characterized by DNA hybridization in this study or in the studies of Feresu belonged to either L. kirschneri or L. santarosai (Table 1; Feresu et al., 1993, 1994, 1995, 1996, 1998, 1999). While these observations are intriguing, additional studies with substantially more strains are necessary to determine their accuracy.

The study of Yasuda et al. (1987) on speciation of leptospires was extended by Ramadass et al. (1992) who used slot-blot hybridization. They described the new species, $L$. kirschneri, with serovar cynopteri strain $3522 \mathrm{C}$ as its type strain. In the present study, all serovars used by Ramadass et al. (1992) were examined, except for serovar sumatrana, which they identified as $L$. interrogans and serovar vietnam, which they identified as $L$. borgpetersenii. Ramadass et al. (1992) did not speciate strains from nine serovars. One of these, serovar nicaragua strain 1011 , was $100 \%$ related to $L$. noguchii according to their data, and we identified it as $L$. noguchii. Six strains, serovar atchafalaya strain LSU 1013, serovar borincana strain HS 622, serovar bravo strain Bravo, serovar gatuni strain $1473 \mathrm{~K}$, serovar luis strain M 6 and serovar rama strain 316, were identified by us as L. santarosai. Four of these gave high, but less than species-level relatedness to $L$. santarosai in the Ramadass et al. (1992) study. Serovar ballum strain Mus 127, which was most highly related to $L$. borgpetersenii in their study, was identified as L. borgpetersenii in our study and that of Yasuda et al. (1987). Serovar szwajizak strain Szwajizak, which was not highly related to any species in their study, was identified as L. interrogans in our study.

Of the 55 other strains identified in the study of Ramadass et al. (1992), their identification of seven strains differed from that obtained in either the present study and/or the study of Yasuda et al. (1987). They identified serovar atlantae strain LT 81 as $L$. interrogans, whereas it was identified as $L$. santarosai by Yasuda et al. (1987) and in our study. We have no explanation for this discrepancy. Ramadass et al. (1992) identified serovar grippotyphosa strain Moskva V as L. kirschneri. This strain was reported as L. interrogans by Yasuda et al. (1987). We confirm its identification as L. kirschneri. This discrepancy resulted from a strain designation error by Yasuda $e t$ al. (1987). They used serovar grippotyphosa strain Andaman, which is $L$. interrogans, but mistakenly reported it as strain grippotyphosa, which is $L$. kirschneri. Another inconsistency, due to an apparent strain designation error, is responsible for a discrepancy in the identification of serovar saxkoebing. Ramadass et al. (1992) reported its reference strain as M 84 and identified it as $L$. borgpetersenii, whereas Yasuda et al. (1987) and in our study, serovar saxkoebing was identified as $L$. interrogans. M 84 is the reference strain for serovar sejroe, not serovar saxkoebing, and serovar sejroe is L. borgpetersenii.

We have not resolved the four remaining inconsistencies. Ramadass et al. (1992) identified serovar dania strain $\mathrm{K} 1$ as $L$. kirschneri; we identified it as $L$. santarosai. They identified serovar muenchen strain München C 90 as $L$. noguchii; we identified it as $L$. santarosai. They identified serovar tunis strain P2/65 as $L$. santarosai; we identified it as $L$. borgpetersenii. They identified serovar worsfoldi strain Worsfold as $L$. weilii; we identified it as $L$. borgpetersenii.

Of the pairs of species involved in these discrepancies, only $L$. weilii and L. borgpetersenii pose any difficulty in differentiation if divergence of related sequences and relatedness at a stringent DNA reassociation $\left(70^{\circ} \mathrm{C}\right)$ are not done. Despite the substantial methodological differences in our study and that of Ramadass et al. (1992) (they used slot-blot hybridization only at $60{ }^{\circ} \mathrm{C}$ ), it is doubtful that differences in hybridization methodology are responsible for the discrepancies.

The taxonomic DNA hybridization methods used in this study require the growth of leptospires in substantial quantity and the use of a radioactive isotope. These requirements limit the utility of these methods to very few laboratories and even then are not suited for routine use in speciation. In recent years, several molecular approaches have been developed for the identification of leptospires at the species and serovar level. These include whole chromosome restriction endonuclease patterns, PCR amplification of 23S rDNA and artibrarily primed DNA fingerprinting (Marshall et al., 1981; Feresu et al., 1994, 1995; Corney et al., 1997; Woo et al., 1997). Where compared, the results obtained using these methods are comparable to those obtained using DNA hybridization. These methods are significantly less resource-intensive and, therefore, more widely applicable. The findings presented in the present study should provide a molecular taxonomic framework for the continued development and application of these new identification approaches.

The five new Leptospira genomospecies identified in this study are described below. Only one of these, Leptospira genomospecies 2 , is formally named, because the other genomospecies presently contain only one or two strains.

\section{Description of Leptospira alexanderi sp. nov. (Leptospira genomospecies 2)}

Leptospira alexanderi (a.lex.an'der.i. N.L. gen. n. alexanderi to honour Aaron D. Alexander, an 
American microbiologist who has devoted more than 40 years to the study of leptospires). The first leptospiral DNA hybridization studies that were conducted in Professor Alexander's laboratory in 1969 and 1974 (Haapala et al., 1969; Brendle et al., 1974) inspired the study of Yasuda et al. (1987) and the present study.

Cells are Gram-negative, flexible and helical. Motile by means of two flagella (axial fibriles). Obligately aerobic and oxidase-positive. $\mathrm{NaCl}$ is not required for growth. No growth at 11 or $37^{\circ} \mathrm{C}$. Growth is inhibited by 8 -azaguanine $\left(225 \mu \mathrm{g} \mathrm{ml}^{-1}\right)$ and 2,6-diaminopurine $\left(10 \mu \mathrm{g} \mathrm{m}^{-1}\right)$. Growth in the presence of copper sulfate (100 p.p.m.) and production of lipase are variable. It contains serovars from serogroup Manhao (serovar lushui and manhao 3), Hebdomadis (serovar manzhuang and nanding), Javanica (serovar mengla) and Mini (serovar yunnan). DNA $\mathrm{G}+\mathrm{C}$ content is $38.0 \mathrm{~mol} \%$. All strains isolated to date are from China. The type strain, L $60^{\mathrm{T}}$ (= ATCC $700520^{\mathrm{T}}$; serovar manhao 3) was isolated in China from an unknown source. DNA relatedness among the strains in the species and their relatedness to other leptospires are shown in Tables 2 and 3.

\section{Description of Leptospira genomospecies 1}

Cells are Gram-negative, flexible and helical. Motile by means of two flagella (axial fibriles). Obligately aerobic and oxidase-positive. $\mathrm{NaCl}$ is not required for growth. No growth at 11 or $37^{\circ} \mathrm{C}$. Growth is inhibited by 8 -azaguanine $\left(225 \mu \mathrm{g} \mathrm{ml} \mathrm{m}^{-1}\right), 2,6$-diaminopurine $\left(10 \mu \mathrm{g} \mathrm{ml}^{-1}\right)$ and copper sulfate. Lipase is not produced. It contains serovars from serogroups Ranarum and an as yet undesignated serogroup. DNA $\mathrm{G}+\mathrm{C}$ content is $39.8 \mathrm{~mol} \%$. The type strain is $79601^{\mathrm{T}}$ (=ATCC $700521^{\mathrm{T}}$; serovar sichuan), isolated in China from a frog. The DNA relatedness relationships of the strains in the species and their relatedness to other leptospires are shown in Tables 2 and 3.

\section{Description of Leptospira genomospecies 3}

Cells are Gram-negative, flexible and helical. Motile by means of two flagella (axial fibriles). Obligately aerobic and oxidase-positive. $\mathrm{NaCl}$ is not required for growth. No growth at 11 or $37^{\circ} \mathrm{C}$. Growth is inhibited by 8 -azaguanine $\left(225 \mu \mathrm{g} \mathrm{ml} \mathrm{m}^{-1}\right)$, 2,6-diaminopurine $\left(10 \mu \mathrm{g} \mathrm{ml} \mathrm{m}^{-1}\right)$ and copper sulfate. Lipase is not produced. It contains serovars from an as yet undesignated serogroup. The type and only strain isolated to date, is WaZ Holland ${ }^{\mathrm{T}}$ ( = ATCC $700522^{\mathrm{T}}$; serovar holland), isolated from water in the Netherlands. DNA G $+\mathrm{C}$ content is $43.4 \mathrm{~mol} \%$. The DNA relatedness relationships to other leptospires are shown in Tables 2 and 3.

\section{Description of Leptospira genomospecies 4}

Cells are Gram-negative, flexible and helical. Motile by means of two flagella (axial fibriles). Obligately aerobic and oxidase-positive. $\mathrm{NaCl}$ is not required for growth. No growth at 11 or $37^{\circ} \mathrm{C}$. Growth is inhibited in the presence of 2,6-diaminopurine $\left(10 \mu \mathrm{g} \mathrm{ml}^{-1}\right)$, but not by 8 -azaguanine $\left(225 \mu \mathrm{g} \mathrm{ml}^{-1}\right)$ or copper sulfate. Lipase is not produced. It contains serovars from serogroup Icterohaemorrhagiae. The type and only strain isolated to date is $\mathrm{H} 2^{\mathrm{T}}$ (= ATCC $700639^{\mathrm{T}}$; serovar hualin), isolated from an unknown source in China. DNA G $+\mathrm{C}$ content is $38.9 \mathrm{~mol} \%$. The DNA relatedness relationships to other leptospires are shown in Tables 2 and 3.

\section{Description of Leptospira genomospecies 5}

Cells are Gram-negative, flexible and helical. Motile by means of two flagella (axial fibriles). Obligately aerobic and oxidase-positive. $\mathrm{NaCl}$ is not required for growth. No growth at 11 or $37^{\circ} \mathrm{C}$. Growth is inhibited in the presence of 2,6-diaminopurine $\left(10 \mu \mathrm{g} \mathrm{ml}^{-1}\right)$, but not by 8 -azaguanine $\left(225 \mathrm{~g} \mathrm{~m}^{-1}\right)$ or copper sulfate. Lipase is not produced. It contains serovars from serogroup Semaranga. The type strain is Sao Paulo ${ }^{\mathrm{T}}$ (= ATCC $700523^{\mathrm{T}}$; serovar saopaulo), isolated in Brazil from water. DNA G $+\mathrm{C}$ content is $37.9 \mathrm{~mol} \%$. The DNA relatedness relationships to other leptospires are shown in Tables 2 and 3.

\section{REFERENCES}

Brendle, J. J., Rogul, M. \& Alexander, A. D. (1974). Deoxyribonucleic acid hybridization among selected leptospiral serotypes. Int J Syst Bacteriol 24, 205-214.

Brenner, D. J. (1991). Taxonomy, classification, and nomenclature of bacteria. In Manual of Clinical Microbiology, 5th edn, pp. 209-215. Edited by A. Balows, W. J. Hausler, Jr, K. L. Herrman, H. D. Isenberg \& H. J. Shadomy. Washington, DC: American Society for Microbiology.

Brenner, D. J., McWhorter, A. C., Leete-Knutson, J. K. \& Steigerwalt, A. G. (1982). Escherichia vulneris: a new species of Enterobacteriaceae associated with human wounds. J Clin Microbiol 15, 1133-1140.

Brenner, D. J., Grimont, P. A. D., Steigerwalt, A. G., Fanning, G. R., Ageron, E. \& Riddle, C. F. (1993). Classification of citrobacteria by DNA hybridization: designation of Citrobacter farmeri sp. nov., Citrobacter youngae sp. nov., Citrobacter braakii sp. nov., Citrobacter werkmanii sp. nov., Citrobacter sedlakii sp. nov., and three unnamed Citrobacter genomospecies. Int $J$ Syst Bacteriol 43, 645-658.

Corney, B. G., Colley, J. \& Graham, G. C. (1997). Simplified analysis of pathogenic leptospiral serovars by random amplified polymorphic DNA fingerprinting. $J$ Med Microbiol 46, 927-932.

Feresu, S. B., Bolin, C. A. \& Korver, H. (1993). A new leptospiral serovar in the Icterohaemorrhagiae serogroup isolated from an ox in Zimbabwe. Int $J$ Syst Bacteriol 43, 179-182.

Feresu, S. B., Bolin, C. A., Korver, H. \& Terpstra, W. J. (1994). Classification of leptospires of the Pyrogenes serogroup isolated from cattle in Zimbabwe by cross-agglutinin absorption and restriction fragment length polymorphism analysis. Int $J$ Syst Bacteriol 44, 541-546. 
Feresu, S. B., Bolin, C. A., Korver, H. \& Van De Kemp, H. (1995). Identification of leptospires of the Pomona and Grippotyphosa serogroups isolated from cattle in Zimbabwe. Res Vet Sci 59 , 92-94.

Feresu, S. B., Konver, H., Riquelme, N., Baranton, G. \& Bolin, C. A. (1996). Two new leptospiral serovars in the Hebdomadis serogroup isolated from Zimbabwe cattle. Int $J$ Syst Bacteriol 46, 694-698.

Feresu, S. B., Bolin, C. A. \& Korver, H. (1998). A new leptospiral serovar, ngavi, in the Tarassovi serogroup isolated from Zimbabwe oxen. Int J Syst Bacteriol 48, 207-213.

Feresu, S. B., Steigerwalt, A. G. \& Brenner, D. J. (1999). DNA relatedness of Leptospira strains isolated from beef cattle in Zimbabwe. Int J Syst Bacteriol (in press).

Haapala, D. K., Rogul, M., Evans, L. B. \& Alexander, A. D. (1969). Deoxyribonucleic acid base composition and homology studies of Leptospires. J Bacteriol 98, 421-428.

Hovind-Hougen, K. (1979). Leptospiraceae, a new family to include Leptospira Noguchi 1917 and Leptonema gen. nov. Int $J$ Syst Bacteriol 29, 245-251.

Hovind-Hougen, K., Ellis, W. A. \& Birch-Andersen, A. (1981). Leptospira parva sp. nov.: some morphological and biological characters. Zentbl Bakteriol Parasitenkd Infektionskr Hyg Abt 1 Orig Reihe A 250, 343-354.

Johnson, R. C. \& Faine, S. (1984). Family II. Leptospiraceae. In Bergey's Manual of Systematic Bacteriology, pp. 62-67. Edited by N. R. Krieg \& J. G. Holt. Baltimore: Williams \& Wilkins.

Kmety, E. \& Dikken, H. (1988). Revised list of Leptospira serovars. I. Alphabetical order. 14 pp. Groningen: University Press.
Kmety, E. \& Dikken, H. (1993). Classification of the species Leptospira interrogans and history of its serovars. 104 pp. Groningen: University Press.

Mandel, M., Igambi, L., Bergendahl, J., Dodson, M. L., Jr \& Scheltgen, E. (1970). Correlation of melting temperature and cesium chloride buoyant density of bacterial deoxyribonucleic acid. $J$ Bacteriol 101, 333-338.

Marshall, R. (1992). International Committee on Systematic Bacteriology Subcommittee on the Taxonomy of Leptospira. Minutes of the Meetings, 13 and 15 September 1990, Osaka, Japan. Int J Syst Bacteriol 42, 330-334.

Marshall, R. B., Wilton, B. E. \& Robinson, A. J. (1981). Identification of leptospiral serovars by restriction endonuclease analysis. J Med Microbiol 14, 163-166.

Ramadass, P., Jarvis, B. D. W., Corner, R. J., Penny, D. \& Marshall, R. B. (1992). Genetic characterization of pathogenic Leptospira species by DNA hybridization. Int J Syst Bacteriol 42, 215-219.

Wayne, L. G., Brenner, D. J., Colwell, R. R. \& 9 other authors (1987). International Committee on Systematic Bacteriology. Report of the ad hoc committee on reconciliation of approaches to bacterial systematics. Int J Syst Bacteriol 37, 463-464.

Woo, T. H., Smythe, L. D., Symonds, M. L., Norris, M. A., Dohnt, M. F. \& Patel, B. K. (1997). Rapid distinction between Leptospira interrogans and Leptospira biflexa by PCR amplification of 23S ribosomal DNA. FEMS Microbiol Lett 150, 9-18.

Yasuda, P. H., Steigerwalt, A. G., Sulzer, K. R., Kaufmann, A. F., Rogers, F. \& Brenner, D. J. (1987). Deoxyribonucleic acid relatedness between serogroups and serovars in the family Leptospiraceae with proposals for seven new Leptospira species. Int J Syst Bacteriol 37, 407-415. 